\title{
Assembly-level analyses of accident-tolerant cladding concepts for a long-life civil marine SMR core using micro-heterogeneous duplex fuel
}

\author{
Syed Bahauddin Alam,*, Cameron S. Goodwin ${ }^{\mathrm{b}}$, Geoffrey T. Parks \\ ${ }^{a}$ Department of Engineering, University of Cambridge \\ Cambridge, CB2 1PZ, United Kingdom \\ ${ }^{b}$ Rhode Island Atomic Energy Commission \\ 16 Reactor Rd, Narragansett, RI 02882, USA
}

\begin{abstract}
In this reactor physics study, we examine the neutronic performance of accident-tolerant fuel (ATF) claddings - austenitic type 310 stainless steel (310SS), ferritic Fe-20Cr-5Al (FeCrAl), advanced powder metallurgic ferritic (APMT), and silicon carbide (SiC)-based materials - as alternative cladding materials compared with Zircaloy-4 (Zr) cladding. The cores considered use $18 \%{ }^{235} \mathrm{U}$ enriched micro-heterogeneous $\mathrm{ThO}_{2}-\mathrm{UO}_{2}$ duplex fuel and, for purposes of comparison, $15 \%{ }^{235} \mathrm{U}$ enriched homogeneously mixed all- $\mathrm{UO}_{2}$ fuel, loaded into $13 \times 13$ pin arrays. A constant cladding coating thickness of $655 \mu \mathrm{m}$ is assumed. We use the WIMS reactor physics code to analyse the associated reactivity, achievable discharge burnup, spectral variations, rim effect and reactivity feedback parameters for the candidate cladding materials at the assembly level.

The results show that candidate fuels with 310SS cladding exhibit a $\sim 13 \%$ discharge burnup penalty compared to $\mathrm{Zr}$ due to the presence of a very high nickel (Ni) concentration. The high neutron absorption cross-sections of iron (Fe) in the FeCrAl and APMT claddings also lead to a $\sim 10 \%$ discharge burnup penalty. The fuels with $\mathrm{SiC}$ cladding can achieve a $\sim 1 \%$ higher discharge burnup compared to Zr due to the low thermal neutron absorption cross-sections of its constituents and the softer neutron spectrum. The claddings with lower capture cross-sections ( $\mathrm{SiC}$ and $\mathrm{Zr}$ ) exhibit higher relative fission power at the pellet periphery. For both candidate fuels, the end-of-life ${ }^{239} \mathrm{Pu}$ (for $\mathrm{UO}_{2}$ fuel) and ${ }^{233} \mathrm{U}$ (for duplex fuel) inventories are higher for the claddings (Fe-based: FeCrAl, APMT and steel-based: 310SS) with higher thermal capture cross-sections, unlike for $\mathrm{SiC}$ and $\mathrm{Zr}$, where $\mathrm{SiC}$ provides higher end-of-life ${ }^{239} \mathrm{Pu}$ and ${ }^{233} \mathrm{U}$ inventories despite having lower capture cross-section than that of the Zr. Reactivity feedback parameter values (moderator and fuel temperature coefficients) are more negative for the duplex fuel than the $\mathrm{UO}_{2}$ fuel for all the candidate claddings, with claddings with harder spectra exhibiting more negative values. The duplex fuel yields a softer spectrum than the $\mathrm{UO}_{2}$ fuel with the candidate claddings, which improves neutron economy and thus discharge burnup.
\end{abstract}

Keywords: Accident-tolerant cladding, Soluble-boron-free design, Micro-heterogeneous duplex fuel, Reactivity, Achievable discharge burnup, Spectral hardening, Rim effect, 


\section{Introduction}

Marine propulsion has been an important application of nuclear energy since the earliest days of power reactors. Nuclear propulsion research was first undertaken in the U.S.A. in the 1940s and has led to significant engineering outcomes, including the invention of the pressurized water reactor (PWR) and the development of important safety practices (Khlopkin and Zotov, 1997, Hirdaris et al., 2014, Vergara and McKesson, 2002). Since the 1955 launch of the USS Nautilus, nuclear naval vessels have accrued over 12,000 reactor-years of operational experience, demonstrating that with effective technology and training, nuclear marine propulsion can be a safe and reliable option (Hirdaris et al., 2014, Ragheb, 2011). The U.S. 'Nuclear Navy' has a record of reliable power with no major radiation releases in the course of 5400 reactor-years of operation (Hirdaris et al., 2014, Vergara and McKesson, 2002). U.S. research establishments, including Bettis and Knolls Atomic Power Laboratories, are continuing to work on the development of naval nuclear propulsion technology.

U.S. naval reactors use very highly enriched uranium fuel, giving very long core lifetimes compared to civil nuclear power plant. Russia has long experience ( $\sim 60$ years) in designing and operating PWR-type reactors for nuclear-powered icebreakers. Historically Russian ships (e.g. OK-900, KLT-40) have utilised a cermet fuel with uranium enriched to more than $20 \%$ ${ }^{235} \mathrm{U}$ (Bukharin, 2006), but a new generation of icebreaker cores (e.g. KLT-40S, RITM-200) are reported to use cermet fuel consisting of zirconium-based alloy host material embedded with $\mathrm{UO}_{2}$ particles enriched to less than $20 \%{ }^{235} \mathrm{U}$ (Zverev et al., 2013). The reactor will have a relatively low maximum power output of $174 \mathrm{MWth}$, a low capacity factor (around $65 \%$ ) and require refueling every 7 years (Bukharin, 2006, Prasad et al., 2015).

In spite of this track record, nuclear propulsion has never played a significant role in the civil maritime sector. There are several interconnected reasons for this, including political barriers created by popular antinuclear sentiment, a lack of infrastructure and institutions supporting nuclear shipping, and the high up-front costs of purchasing a nuclear vessel (Dedes et al., 2011, Kramer, 1962). Finally, there are considerable technical and engineering challenges unique to civilian marine reactors (Carlton et al., 2011, Aspelund et al., 2006, Schinas and Stefanakos, 2012), which must be capable of operating with high reliability and little maintenance in a demanding seaborne environment and of achieving long life with low fissile loading (less than $20 \%$ enrichment). Meeting these engineering objectives will require a sustained commitment to research and development.

The US Navy Nuclear Propulsion program began to employ zircaloy cladding in the nuclear-powered submarine USS Nautilus in 1951, due to its favourable metallurgical and neutronic characteristics, including excellent thermal conductivity, its ability to withstand heavy neutron irradiation, and lower thermal neutron absorption cross-section (Azevedo,

\footnotetext{
*Corresponding author

Email address: sba26@cam.ac.uk (Syed Bahauddin Alam)
} 
2011, Terrani et al., 2014). However, neutron irradiation adversely affects the mechanical stability and thermal properties of Zr cladding. There is accumulation of significant corrosion growth and the risk of failure by ballooning and burst in the cladding at temperatures above $\sim 1100 \mathrm{~K}$. As the core temperature increases, Zr exhibits poor oxidation qualities in the high-temperature steam environment, accelerating core degradation processes. Thus, there are clear incentives to investigate alternative accident-tolerant fuel (ATF) cladding materials that will ideally exhibit higher thermal conductivity, a much slower oxidation rate, reduced hydrogen generation, and improvements in the integrity of fission product barriers in a long-life, high-power-density (HPD) PWR marine core (George et al., 2015). Advanced ATF cladding materials often have high neutron capture cross-sections, which are detrimental to the neutron economy and hence core life. It is therefore important to assess the neutronic properties of ATF cladding for our target application. The replacement of Zr cladding with advanced ATF cladding is necessary to ensure the ultra-high safety of our proposed HPD $\left(100 \mathrm{MW} / \mathrm{m}^{3}\right)$ and 15 effective full-power-years life (at least) soluble-boron-free (SBF) PWR marine small modular reactor (SMR) core (Alam, 2018, Alam et al., 2018a,b).

All previous accident-tolerant cladding concept studies have been limited to uraniumbased fuel with soluble boron system (for reactivity control) (Azevedo, 2011, Terrani et al., 2014, George et al., 2015, Pint et al., 2013). In a recent study (George et al., 2015), a neutronic analysis of candidate alternate cladding ATF in a PWR environment was performed by Oak Ridge National Laboratory (ORNL). However, this neutronic study was limited to a single $\mathrm{UO}_{2}$ fuel pin. Other recent studies (Wu et al., 2015, Terrani et al., 2014, Pint et al., 2013) performed neutronics and fuel performance assessments of the ATF concept, which was limited only to advanced oxidation-resistant iron-based claddings with $\mathrm{UO}_{2}$ fuel. There is also a recent study (Naceur and Marleau, 2018) conducted by École Polytechnique de Montréal on potential accident tolerant cladding concepts in a CANDU-6 (Canada Deuterium Uranium) reactor, where the analyses were also limited to the $\mathrm{UO}_{2}$ fuel only. Furthermore, a Brookhaven National Laboratory team performed an extensive screening of advanced ATF concepts and neutronic evaluations of $\mathrm{UN}-\mathrm{U}_{3} \mathrm{Si}_{5}$ relative to the $\mathrm{UO}_{2}$ reference fuel with ATF claddings (Brown et al., 2015). Apart from the advanced ATF concepts, a neutronic evaluation of ceramic coatings for the PWR and BWR systems with the $\mathrm{UO}_{2}$ fuel was performed (Younker and Fratoni, 2016) jointly by the Pennsylvania State University and the University of California, Berkeley. In addition, an assessment on advanced stainless steel and silicon carbide for $\mathrm{UN}$ and $\mathrm{U}_{3} \mathrm{Si}_{2}$ fuels for the Integral Inherently Safe Light Water Reactor (I2S-LWR) (Lindley et al., 2016) was conducted by Amec Foster Wheeler. It can, therefore, be confirmed from the published works on neutronic assessment of ATF concepts that although appreciable amounts of work on ATF have been performed, all previous accident-tolerant cladding concept studies have been limited to uranium-based fuel, and the feasibility of these claddings has not been observed for SBF system and long-life SMR core. Furthermore, there is a significant gap in assessing the ATF concepts for the thorium-based fuel and previously no ATF analysis has been performed for the exotic micro-heterogeneous duplex fuel concept. In this regard, it is indeed necessary to perform this ATF analyses for the SBF, SMR and thorium-based micro-heterogeneous duplex fuel concept.

In addition, previous reactor physics and core design studies have indicated that ho- 
mogeneously mixed $\mathrm{Th} / \mathrm{UO}_{2}$ fuel only offers promising performance in a single-batch core when the ${ }^{235} \mathrm{U}$ enrichment exceeds $20 \%$ (Galperin et al., 2002, Otto, 2013) and thorium's advantages are best realized in micro-heterogeneous and heterogeneous geometries (Kazimi et al., 1999, Todosow et al., 2005, Clayton, 1993, Otto, 2013, MacDonald and Lee, 2004). However, heterogeneous seed-blanket arrangements rely on being able to remove the seed region and replace it mid-life with fresh fuel (Kazimi et al., 1999, Todosow et al., 2005, Clayton, 1993), which is not compatible with single-batch SMR operation. The ability of the micro-heterogeneous duplex fuel to exploit the potential benefits of thorium in the context of a single-batch, low enriched uranium, SBF, long-life, SMR core has yet to be fully explored (Zhao, 2001, MacDonald and Lee, 2004). This motivates the assessment of the reactor physics behaviour of radially micro-heterogeneous $\mathrm{ThO}_{2}-\mathrm{UO}_{2}$ duplex fuel ${ }^{1}$ with the candidate claddings in a SBF environment, loaded in a single-batch strategy. To provide a basis for comparison we also evaluate the performance of homogeneously mixed all- $\mathrm{UO}_{2}$ fuel. In this paper, higher fissile loading than the current practice (for ensuring high burnup/long core life) is utilized in the proposed SBF, SMR core and therefore, it is required to observe the behaviour of the ATF claddings with the duplex fuel for this SBF and high burnup SMR application.

Several advanced ATF claddings that can withstand a loss-of-coolant accident (LOCA) for a considerably longer time period than current PWR Zr cladding are assessed. Austenitic and ferritic alloys and silicon carbide ( $\mathrm{SiC}$ ) cladding have therefore been considered, assuming a constant cladding coating thickness of $655 \mu \mathrm{m}$, for a single PWR marine assembly. These cladding materials have the ability to significantly reduce oxidation kinetics in hightemperature steam environments when compared to Zr alloys, which in turn could reduce heating and hydrogen generation rates. A reactor physics assessment in terms of reactivity, achievable discharge burnup, spectral variations, rim effect and reactivity feedback parameters associated with various candidate ATF claddings is undertaken in comparison with the reference $\mathrm{Zr}$ cladding for a long-life marine PWR core using $18 \%{ }^{235} \mathrm{U}$ enriched duplex fuel and $15 \%{ }^{235} \mathrm{U}$ enriched homogeneously mixed all- $\mathrm{UO}_{2}$ fuel.

\section{Candidate cladding materials}

In an environment of high-temperature steam with poor heat transfer to the gaseous phase, Zr cladding will exhibit self-catalytic oxidation (Terrani et al., 2014). The enthalpy production from the oxidation reaction, along with decay heat, will cause the temperature of the fuel to continue to rise towards its melting point. Accident-tolerant cladding can enable fuel to tolerate the loss of active cooling for a significantly longer period. Cladding with improved oxidation resistance and reduced heat and/or hydrogen generation is expected to achieve larger margins of safety in severe accident scenarios. In this study, the neutronic performance of alternative non-Zr cladding materials is investigated and compared to $\mathrm{Zr}$ alloy cladding. The materials considered are: austenitic stainless steel type 310SS; two ferritic alloys: FeCrAl and an advanced powder metallurgic ferritic alloy (APMT); and SiC.

\footnotetext{
${ }^{1}$ We use the term 'duplex' to refer to micro-heterogeneous $\mathrm{ThO}_{2}-\mathrm{UO}_{2}$ duplex fuel throughout this paper.
} 
Austenitic 310SS cladding has higher chromium $(\mathrm{Cr})$ and nickel $(\mathrm{Ni})$ content and exhibits high-temperature steam oxidation resistance as a result of protective surface oxide or scale formed via $\mathrm{Cr}_{2} \mathrm{O}_{3}$ under these conditions (Pint et al., 2013). The high $\mathrm{Ni}, \mathrm{Cr}$ and Fe content in 310SS cladding is responsible for its high neutron absorption properties, which can have a detrimental effect on core life.

Iron (Fe)-based claddings (ferritic alloy FeCrAl and commercial APMT) contain significant proportions of aluminium (Al) and therefore form protective alumina $\left(\mathrm{Al}_{2} \mathrm{O}_{3}\right)$ surface scale (George et al., 2015). Like $\mathrm{Cr}_{2} \mathrm{O}_{3}$, protective alumina scale is less permeable than $\mathrm{ZrO}_{2}$ and contributes to the slower rate of oxidation under high-temperature steam oxidation conditions. It is thus considered safer under accident scenarios. FeCrAl and APMT were examined in this study due to their high strength compared to $\mathrm{Zr}$ alloys.

The study of $\mathrm{SiC}$ is a research effort by the U.S. Department of Energy (DOE) Fuel Cycle Research and Development Advanced Fuels Campaign to develop novel fuel and cladding concepts to replace the current $\mathrm{Zr}$ alloy- $\mathrm{UO}_{2}$ fuel system. Zr-based cladding can experience unfavourable reactions with reactor coolants, such as water, which can cause degradation of the material's ductility and loss of material under normal operating conditions (Azevedo, 2011). It can undergo phase transition, loss of strength, exothermic reaction with steam in a light water reactor, and hydrogen production during LOCA conditions. Current research (Pint et al., 2013) suggests that SiC possesses numerous potential benefits over Zr-based alloys, including remarkable irradiation stability, superior oxidation resistance and reduced hydrogen generation under steam attack (off-normal conditions), as well as increased corrosion resistance (George et al., 2015). These advantages allow SiC cladding to reach high burnup (suitable for long-life cores) under normal operating conditions, as well as providing benefits in accident scenarios.

Tables 1 and 2 show the cladding materials examined in this study with their density and microscopic thermal neutron absorption cross-section $\left(\sigma_{a}\right)$, and detailed elemental compositions (wt \%).

\begin{tabular}{|l|c|c|}
\hline Material & $\begin{array}{c}\text { Density } \\
\left(\mathrm{g} / \mathrm{cm}^{3}\right)\end{array}$ & $\begin{array}{c}\sigma_{a} \\
(\text { barns })\end{array}$ \\
\hline SiC & 2.58 & 0.086 \\
\hline Zircaloy & 6.56 & 0.20 \\
\hline FeCrAl & 7.1 & 2.43 \\
\hline APMT & 7.3 & 2.47 \\
\hline 310SS & 8.03 & 3.21 \\
\hline
\end{tabular}

Table 1. Density and $\sigma_{a}$ for cladding materials (George et al., 2015, George, 2015, Alam, 2018)

It is also worthwhile addressing why the high alloy FeCrAl and APMT are chosen at the same time as candidate claddings. APMT consists of molybdenum (Mo) (2.8 wt\%), unlike $\mathrm{FeCrAl}$, as shown in Table 2. Mo isotopes exhibit resonance behaviour at $\sim 10-100 \mathrm{eV}$ (Brown 


\begin{tabular}{|l|l|c|c|c|c|c|c|c|c|c|c|c|}
\hline Material & $\mathrm{Fe}$ & $\mathrm{Cr}$ & $\mathrm{Al}$ & $\mathrm{Zr}$ & $\mathrm{Ni}$ & $\mathrm{Sn}$ & $\mathrm{Mn}$ & $\mathrm{Mo}$ & $\mathrm{Y}$ & $\mathrm{Si}$ & $\mathrm{Hf}$ & $\mathrm{C}$ \\
\hline SiC & & & & & & & & & & 70.08 & & 29.92 \\
\hline Zircaloy & 0.15 & 0.1 & & 98.36 & & 1.49 & & & & & & \\
\hline FeCrAl & 75 & 20 & 5 & & & & & & & & & \\
\hline APMT & 69.79 & 21.6 & 4.9 & 0.1 & & & & 2.8 & 0.12 & 0.53 & 0.16 & \\
\hline 310SS & 55.55 & 25.22 & & & 19.51 & & 1.9 & 0.122 & & & & \\
\hline
\end{tabular}

Table 2. Candidate cladding materials with their detailed elemental compositions (wt \%) (George et al., 2015, George, 2015, Alam, 2018)

et al., 2015), which is responsible for the significant neutron capture in APMT compared to that of the FeCrAl. Furthermore, due to the presence of Mo, APMT is expected to exhibit different spectral, capture and rim behaviour, which would be of interest to investigate. Both the FeCrAl and APMT have been considered, not based on the $\mathrm{Cr}$ alloy, but to observe the neutronic properties while having the same portions of $\mathrm{Cr}(20 \%$ in $\mathrm{FeCrAl}$ vs. $21.6 \%$ in APMT) in both the APMT and FeCrAl and the presence of Mo in APMT.

\section{Design methods}

\subsection{Rationale behind the selection of the duplex fuel}

While designing the core with the thorium-based fuel, the most important design goal is to optimize the neutron spectrum and increase the conversion of fertile isotopes (conversion of ${ }^{232} \mathrm{Th}$ into ${ }^{233} \mathrm{U}$ ) in the fuel. There are three main strategies to implement thorium fuel: homogeneous mixtures, heterogeneous arrangements, and micro-heterogeneous arrangements (Otto, 2013). The simplest strategy to employ thorium fuel is a homogeneous $\mathrm{Th} / \mathrm{UO}_{2}$ mixture. According to the study conducted by Galperin et al. (Galperin et al., 2002), homogeneously mixed $\mathrm{Th} / \mathrm{UO}_{2}$ fuel exhibits unfavourable neutronic results in terms of burnup/core life. They conclude that single-batch discharge burnup was less than in all-uranium fuel with the same fissile loading for proliferation-compliant $\mathrm{Th} / \mathrm{UO}_{2}$ designs (i.e., ${ }^{235} \mathrm{U} / \mathrm{U}_{\text {total }}<20 \%$ ). Homogeneously mixed $\mathrm{Th} / \mathrm{UO}_{2}$ fuel only shows promising performance in a single batch core when ${ }^{235} \mathrm{U}$ enrichment exceeds $20 \%$ (Galperin et al., 2002, Alam, 2018, Otto, 2013), which isn't compatible with the requirements for our commercial marine core design. The second strategy uses a heterogeneous concept based on seed-and-blanket (SB) approach (Kazimi et al., 1999, Todosow et al., 2005, Clayton, 1993). In this arrangement, the fissile seed region releases neutrons that trigger the conversion of fertile isotopes in the blanket region. It is important to note that cores and assemblies with heterogeneous seed/blanket geometries depend mainly on being able to remove the seed region and replace it mid-life with fresh fuel (Otto, 2013, Alam, 2018). This also isn't compatible with the requirements of single-batch operation for our commercial marine core design. The final strategy is a micro-heterogeneous concept, which is a compromise position between homogeneous and heterogeneous approaches and can be achieved in micro-heterogeneous $\mathrm{ThO}_{2}-\mathrm{UO}_{2}$ duplex fuel. In this concept, the uranium and thorium components are not blended and are discretely interspersed on very small distance scales (Zhao, 2001, Shwageraus et al., 2004, Otto, 2013). Studies (Zhao, 
2001, Shwageraus et al., 2004, Alam, 2018) found that the spatial separation between the fissile ${ }^{235} \mathrm{U}$ and the fertile thorium enables better moderation of the fission neutrons before they interact with the thorium. This spectral shift encourages neutron absorption in the thorium component of the fuel at the beginning of life, helps controlling reactivity and promotes breeding of new fissile material ${ }^{233} \mathrm{U}$. Thorium's advantages are best realized in micro-heterogeneous $\mathrm{ThO}_{2}-\mathrm{UO}_{2}$ and heterogeneous seed-blanket geometries (MacDonald and Lee, 2004), although, as discussed, heterogeneous arrangements will not be considered in this study. A limited number of studies on duplex fuel are available in the public domain, but its use has never been examined in the context of a SBF environment for long-life core (Zhao, 2001, Shwageraus et al., 2004, Alam, 2018, MacDonald and Lee, 2004). Therefore, this study will utilize duplex fuel for the ATF analysis.

\subsection{Computational methods}

The subassembly analysis employed the WIMS-10 lattice physics code using nuclear data from the JEF 2.2 database available from the IAEA (Newton et al., 2008). For each burnup step, WIMS completes a 172-group 'fine' solution to the transport equation in a smeared geometry. It then refines this solution using a few-group calculation in a precise geometry. In this study, we used a 6 energy group structure, as shown in Table 3.

\begin{tabular}{|l|c|c|c|c|c|c|}
\hline Group & 1 & 2 & 3 & 4 & 5 & 6 \\
\hline Upper fine group & 1 & 23 & 46 & 93 & 136 & 153 \\
Lower fine group & 22 & 45 & 92 & 135 & 152 & 172 \\
Upper (eV) & $19.64 \times 10^{6}$ & $820.85 \times 10^{3}$ & $9.12 \times 10^{3}$ & 4.00 & $625 \times 10^{-3}$ & $140 \times 10^{-3}$ \\
Lower (eV) & $820.85 \times 10^{3}$ & $9.12 \times 10^{3}$ & 4.00 & $625 \times 10^{-3}$ & $140 \times 10^{-3}$ & $110 \times 10^{-6}$ \\
\hline
\end{tabular}

Table 3. 6-group WIMS energy structure.

The resonance treatment uses the PRES module to set up the subgroup cross-sections for the nuclides of interest at the fuel temperature and uses the CACTUS module to calculate the subgroup fluxes by the method of characteristics. Finally, the RES module completes the subgroup calculation of resonance shielding.

\subsection{Design of fissile loading}

In our case, an individual fuel pin is composed of a uranium centre surrounded by an annulus of pure thorium in $\mathrm{ThO}_{2}-\mathrm{UO}_{2}$ duplex fuel, as shown in Fig. 1a.

It is worthwhile addressing that it will be many years before civil marine propulsion achieves widespread commercialisation, and rapid improvements in materials and fabrication can lead to permitting ever-higher burnup (Alam, 2018, Otto, 2013). For the purpose of this study, it is assumed that suitable materials and technology that can withstand prolonged cycles and high burnups will be available for future use (Zainuddin, 2015, Sukjai and Kazimi, 2015, Andrews et al., 2014, Otto, 2013). Therefore, higher discharge burnup is not considered as a constraint for the design of long life core, instead, it is an objective. It was assumed in the sizing analysis that the irradiation tolerance of the fuel (100 GWd/tonne) is the primary limiting factor in the core design. 


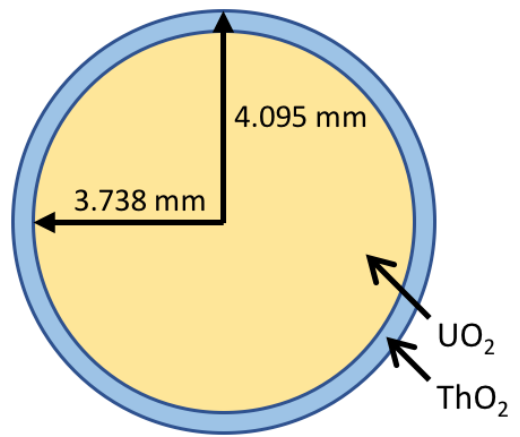

(a)

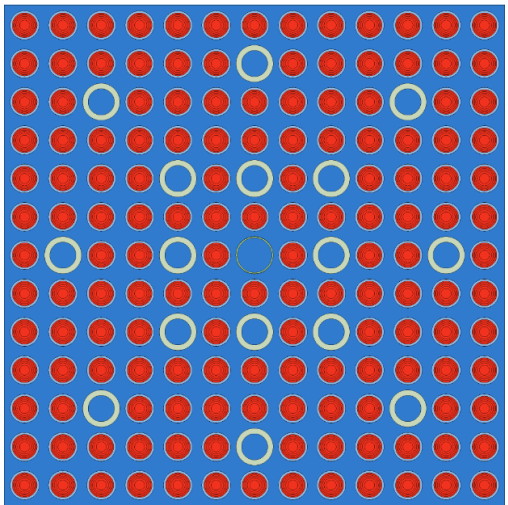

(b)

Fig. 1. (a) Configuration of the micro-heterogeneous duplex $\mathrm{ThO}_{2}-\mathrm{UO}_{2}$ fuel; (b) $13 \times 13$ assembly geometry layout.

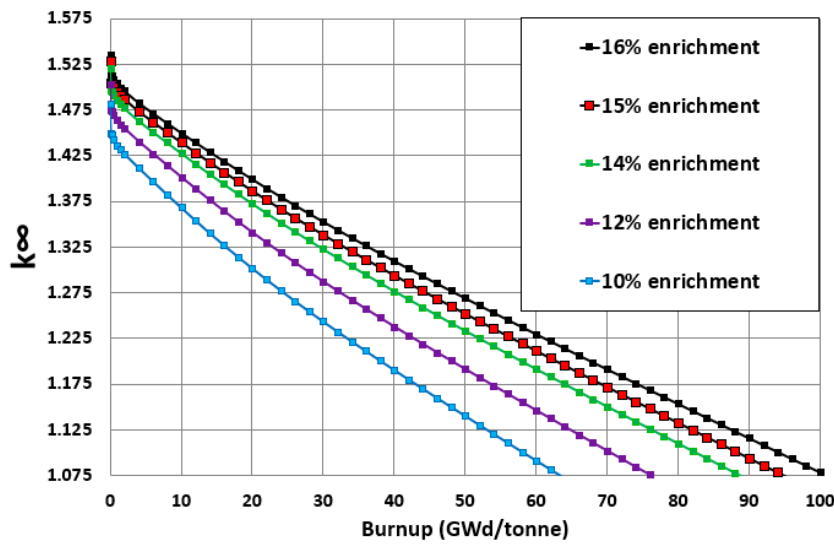

(a)

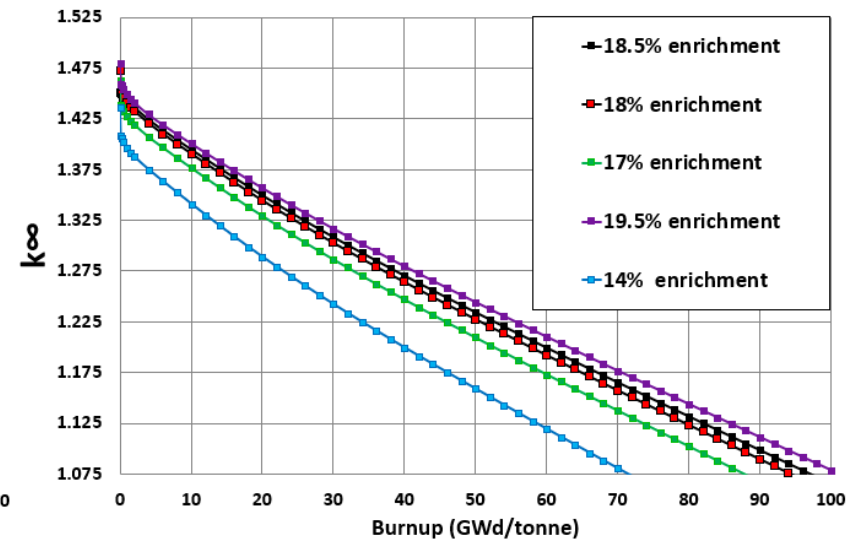

(b)

Fig. 2. Core depletion calculations for various fissile loadings: (a) $\mathrm{UO}_{2}$ fuel; (b) Duplex fuel. 
WIMS calculations assume an infinitely-large core. Since a small core is prone to larger leakage, we have assumed $7.5 \%$ leakage in this study (Alam, 2018). The discharge burnup is therefore estimated from the point on the assembly burnup curve where the infinite multiplication factor, $k_{\infty}$, is 1.075 .

The fissile loadings of the duplex and $\mathrm{UO}_{2}$ fuels were determined from enrichment sensitivity studies for our poison-free SBF $13 \times 13$ assembly (as shown in Fig. 1b), seeking values that keep the core critical for a burnup of $\sim 95 \mathrm{GWd} /$ tonne. It is clear from Figs. $2 \mathrm{a}$ and $2 \mathrm{~b}$ that, in order to achieve the desired discharge burnup, an initial enrichment of $15 \%$ and $18 \%{ }^{235} \mathrm{U}$ will be required for the $\mathrm{UO}_{2}$ and duplex fuels, respectively. The duplex fuel requires higher enrichment than the all- $\mathrm{UO}_{2}$ fuel, in part, due to the lower volume of $\mathrm{UO}_{2}$ in the fuel and, in part, to the higher thermal absorption cross-section of the fertile ${ }^{232} \mathrm{Th}$.

Lattice physics calculations for the assemblies were performed in a previous study (Alam, 2018) using the deterministic transport code WIMS, the Monte Carlo (MC) code Serpent (Leppänen and Pusa, 2009) and the hybrid MC code MONK (Long et al., 2015). For both fuels, excellent agreement $(\sim 100-350 \mathrm{pcm})$ was observed between the codes, providing reassurance that WIMS can be used to generate reliable lattice physics results for SBF marine propulsion cores at much reduced computational cost compared to the MC code Serpent and hybrid MC code MONK.

The design parameters of the proposed marine core are shown in Table 4.

\begin{tabular}{|l|r|}
\hline Parameter & Value \\
\hline Thermal power (MWth) & 333 \\
\hline Minimum desired lifetime (years) & 15 \\
\hline Assembly size & $13 \times 13$ \\
\hline Control rods per assembly & 16 \\
\hline Pin pitch (mm) & 12.65 \\
\hline Fuel pellet diameter (mm) & 8.19 \\
\hline Cladding thickness (mm) & 0.655 \\
\hline Gap thickness (mm) & 0.0498 \\
\hline
\end{tabular}

\begin{tabular}{|l|r|}
\hline Parameter & Value \\
\hline Number of assemblies & 112 \\
\hline Fuel height $(\mathrm{m})$ & 1.79 \\
\hline Core diameter $(\mathrm{m})$ & 1.97 \\
\hline Pitch/diameter ratio & 1.33 \\
\hline Hydrogen-to-heavy metal ratio & 3.99 \\
\hline Assembly side length $(\mathrm{cm})$ & 16.45 \\
\hline Power density $\left(\mathrm{MW} / \mathrm{m}^{3}\right)$ & 63 \\
\hline Average linear rating $(\mathrm{kW} / \mathrm{m})$ & 10 \\
\hline
\end{tabular}

Table 4. Design parameters of proposed marine core (Alam, 2018).

\section{Reactivity}

In an effort to understand the neutronic behaviour of the candidate cladding materials, reactivity calculations were performed for the poison-free SBF $13 \times 13$ assembly. Fig. 3 shows $k_{\infty}$ vs. burnup for the various cladding material candidates with the $\mathrm{UO}_{2}$ and duplex fuels, respectively. Figs. 3a and 3b show that candidate fuels with $\mathrm{SiC}$ cladding achieve the highest discharge burnup $\left(B_{D}\right)$, while all the other ATF claddings fall well short of Zr cladding. Due to the presence of a strong thermal absorber $\left({ }^{232} \mathrm{Th}\right)$, the beginning-of-life (BOL) $k_{\infty}$ of duplex fuel is $\sim 4 \%$ less than for the all- $\mathrm{UO}_{2}$ fuel with the candidate claddings, which is beneficial from the perspective of reactivity control for SBF operation. 


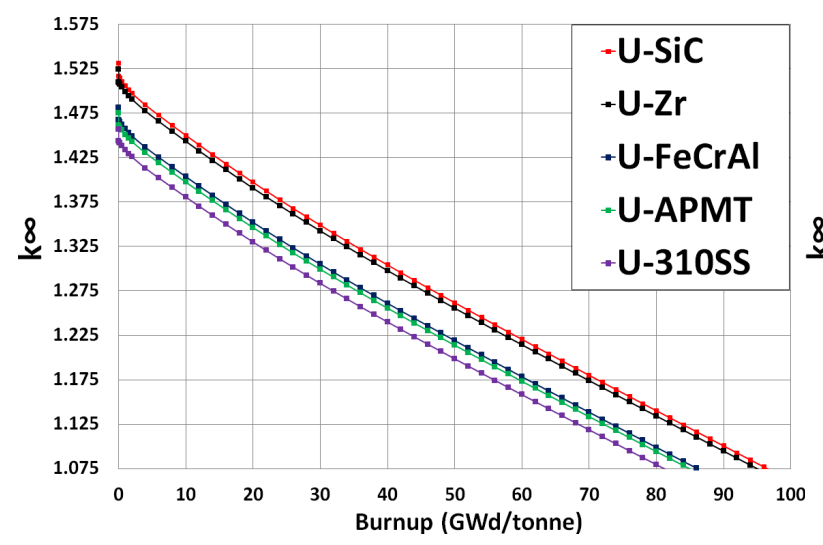

(a)

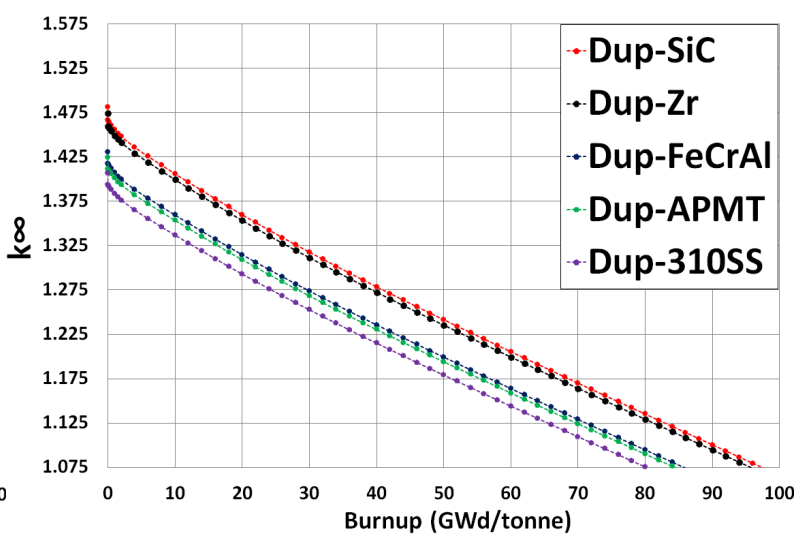

(b)

Fig. 3. $k_{\infty}$ vs. burnup for candidate claddings: (a) $\mathrm{UO}_{2}$ fuel; (b) Duplex fuel.

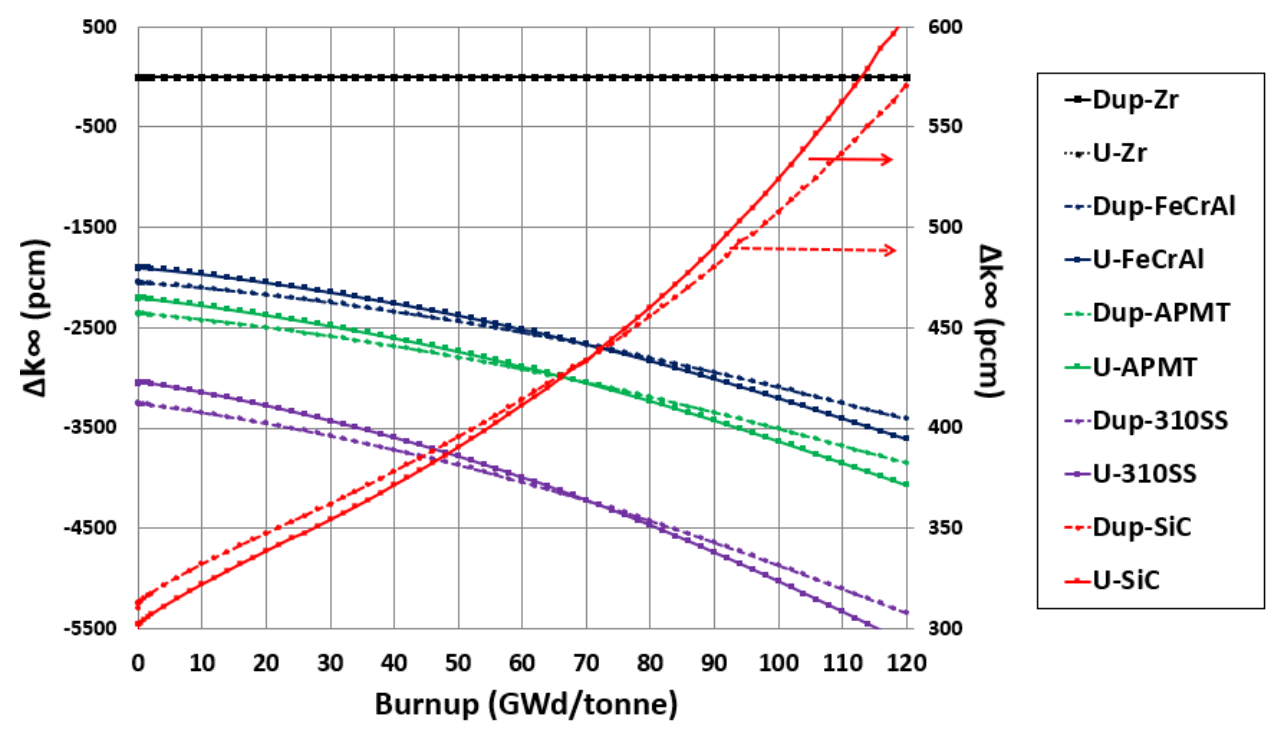

Fig. 4. $\Delta k_{\infty}$ of Zircaloy-4 clad fuel vs. burnup for candidate claddings. 
The effect of the neutron capture cross-section of the alternative claddings on the achievable $B_{D}$ penalty/gain for both candidate fuels can be observed in Fig. 4, where the difference in reactivity from the reference case is shown individually for both the candidate fuels. For both fuels, SiC outperforms $\mathrm{Zr}$ cladding due to its lower thermal neutron capture cross-section and the additional neutron moderation provided by its carbon atoms, which aids in achieving higher BOL $k_{\infty}$ and higher $B_{D}$ compared to Zr. FeCrAl and APMT exhibit $B_{D}$ penalties of $\sim 1 \%$ and $\sim 3 \%$, respectively, compared to the reference $\mathrm{Zr}$, due to the presence of Fe, which has a 12-16 times higher thermal neutron capture cross-section than that of Zr (Fig. 5a). APMT shows a higher burnup penalty than FeCrAl due to the presence of Mo, which has a high resonance absorption cross-section of $\sim 10^{3}-10^{4}$ barns in the resonance energy range (Figs. 5a and 5b). 310SS consists of Fe, a smaller fraction of Mo and a very high Ni concentration. Ni has a thermal neutron capture cross-section, which is about twice that of Fe (Fig. 5a), and 310SS cladding therefore suffers the highest $B_{D}$ penalty.

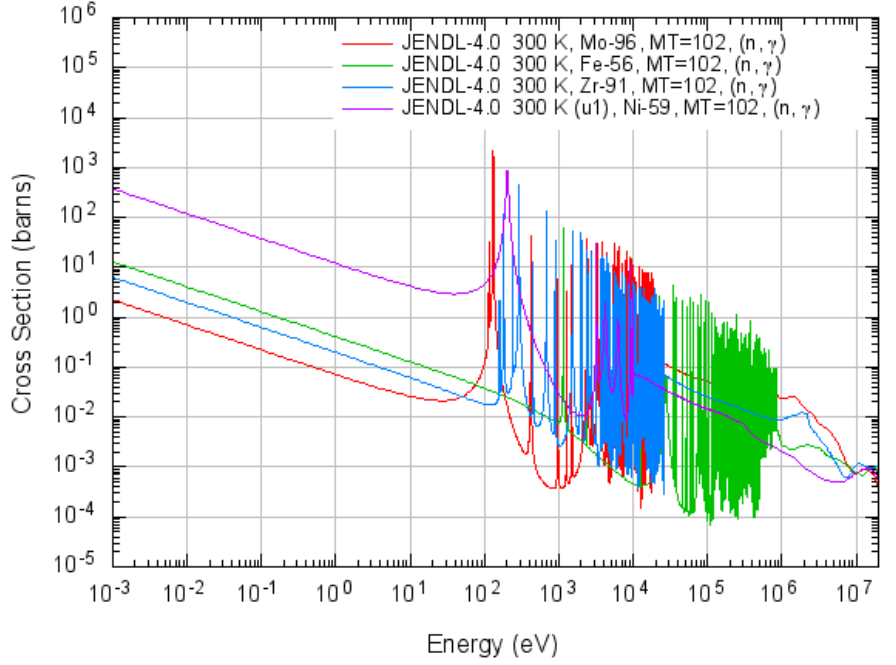

(a)

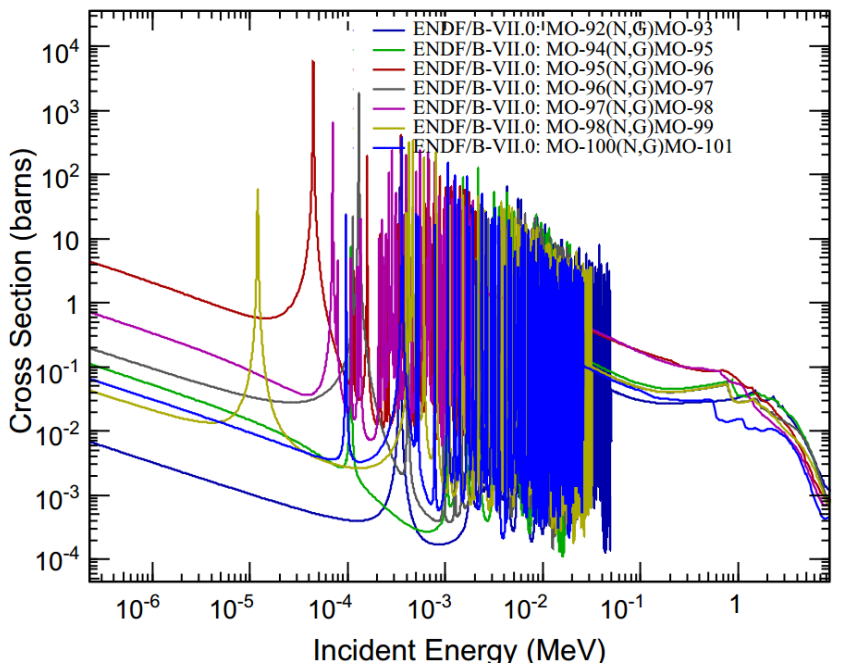

(b)

Fig. 5. Neutron capture cross-sections (a) Key isotopes in candidate claddings (Shibata et al., 2011); (b) Various molybdenum isotopes (Brown et al., 2015).

Fig. 6 illustrates normalized total capture rates in cladding materials per unit lethargy at BOL. Capture rates per unit lethargy are normalized to the 'total capture rates' in cladding materials for the two candidate fuels (Alam, 2018). The total capture in cladding materials refers to the sum of the capture contribution of each elemental compositions of the respective candidate cladding materials. For the candidate fuels (Figs. 6a and 6b), 310SS, APMT and $\mathrm{FeCrAl}$ all exhibit a relatively wide resonance at $0.1 \mathrm{eV}$ (in addition to several narrow resonances at higher neutron energy), which contributes a large fraction of the total neutron captures in these materials, when compared to $\mathrm{Zr}$ and $\mathrm{SiC}$. It can also be seen that APMT cladding exhibits a resonance peak at $\sim 40 \mathrm{eV}$ due to the presence of Mo.

The normalized total cladding capture ratio per unit lethargy was evaluated as the ratio of the total cladding capture rate with duplex fuel to that with $\mathrm{UO}_{2}$ fuel. Fig. 7 shows that the 


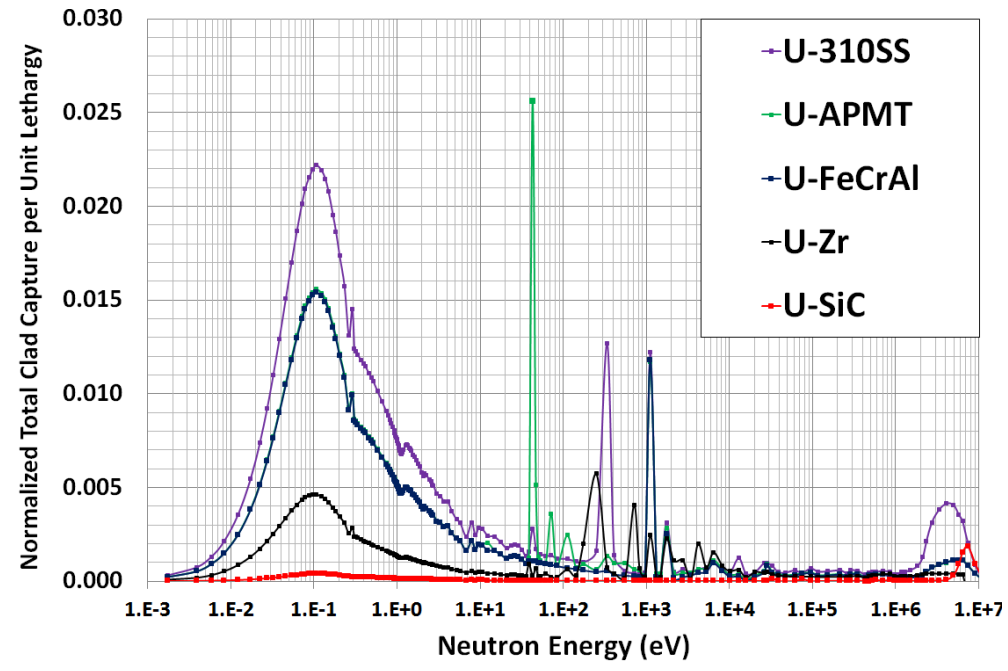

(a)

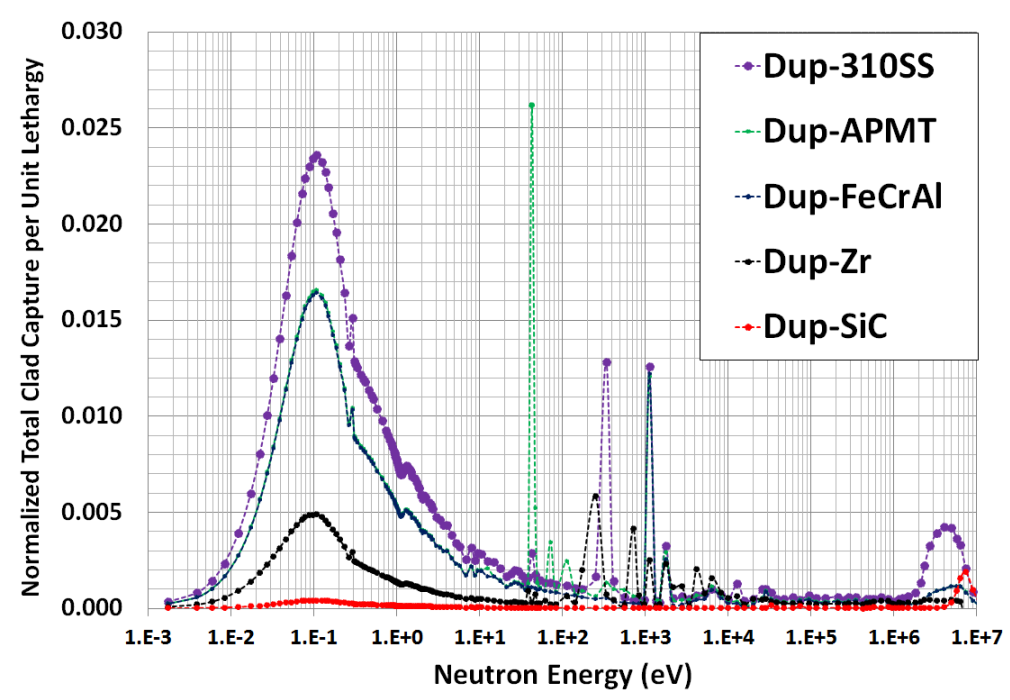

(b)

Fig. 6. Normalized total capture rates in cladding materials per unit lethargy at BOL: (a) $\mathrm{UO}_{2}$ fuel; (b) Duplex fuel. 


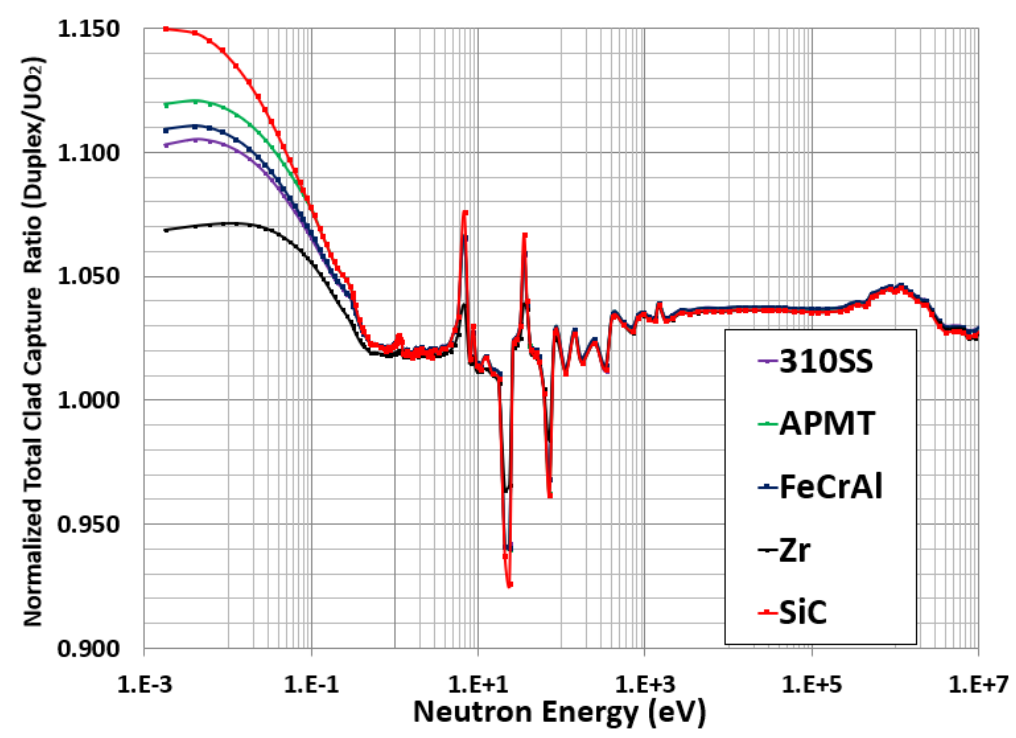

Fig. 7. Normalized total cladding capture ratio (duplex: $\mathrm{UO}_{2}$ ) per unit lethargy at BOL.

cladding capture ratio is above 1.0 over the energy range, suggesting that cladding capture is higher in duplex fuel than $\mathrm{UO}_{2}$ fuel for all of the ATF claddings (Alam, 2018). It can also be observed that duplex fuel contributes to $\sim 5-10 \%$ and $\sim 2-3 \%$ higher cladding capture than $\mathrm{UO}_{2}$ fuel with the candidate claddings in the thermal and fast energy ranges, respectively. Fig. 7 also shows that different cladding candidates exhibit different cladding capture ratios at low energies $(<0.1 \mathrm{eV})$. This originates from the different magnitude of cladding captures with the candidate fuel (mathematical ratio of the total clad capture between duplex to $\mathrm{UO}_{2}$ ) (Alam, 2018). The interference of the fertile resonances of duplex fuel (simultaneous presence of ${ }^{238} \mathrm{U}$ and ${ }^{232} \mathrm{Th}$ ) with the resonances of cladding isotopes (resonance overlap) can lead to a higher overall cladding capture rate for duplex fuel than for $\mathrm{UO}_{2}$ fuel (Zhao, 2001, Shwageraus et al., 2004). Fig. 7 also shows the resonance structure between $\sim 10$ to $\sim 100 \mathrm{eV}$ generated from the absorption resonances of the fissile-fertile combination and ATF claddings (Alam, 2018).

We conclude, therefore, that the replacement of $\mathrm{Zr}$ cladding with $\mathrm{SiC}$ will provide $k_{\infty}$ and $B_{D}$ benefits. The $B_{D}$ penalty associated with the other candidate claddings necessarily shortens the core life, which is detrimental to our design objective of achieving a long-life marine core.

It is also worthwhile addressing that the objective of the SMR, SBF marine propulsion core design was to obtain at least 15 years core life for both candidate fuel cores in the whole-core environment. It has been observed in our study (Alam, 2018) that duplex fuel contributes to $\sim 5 \%$ longer core life than that of the $\mathrm{UO}_{2}$ fuel.

\section{Spectral hardening}

The neutron spectrum is determined by the balance between neutron moderation and absorption. Fig. 8 shows the neutron flux per unit lethargy normalized to unit total flux for the 
candidate claddings. The spectra are plotted in the WIMS 172-group energy structure. The cladding materials containing more absorbing isotopes (higher thermal capture cross-sections) yield a harder neutron spectrum, which is evident from the details of the thermal peaks shown in the top left corners of Figs. 8a and $8 \mathrm{~b}$ for the $\mathrm{UO}_{2}$ and duplex fuels, respectively.

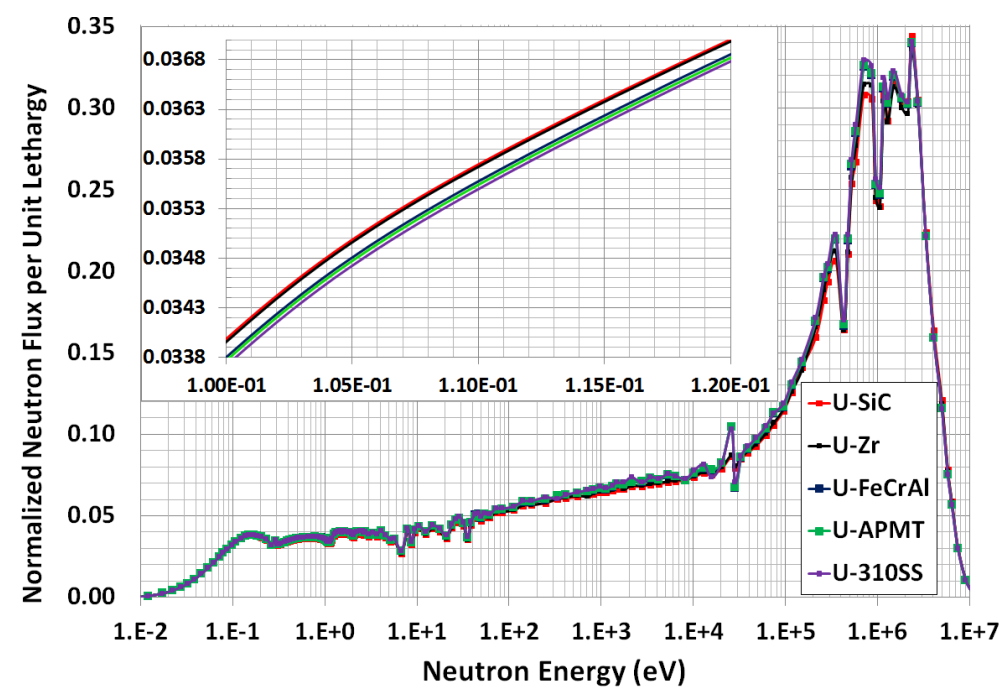

(a)

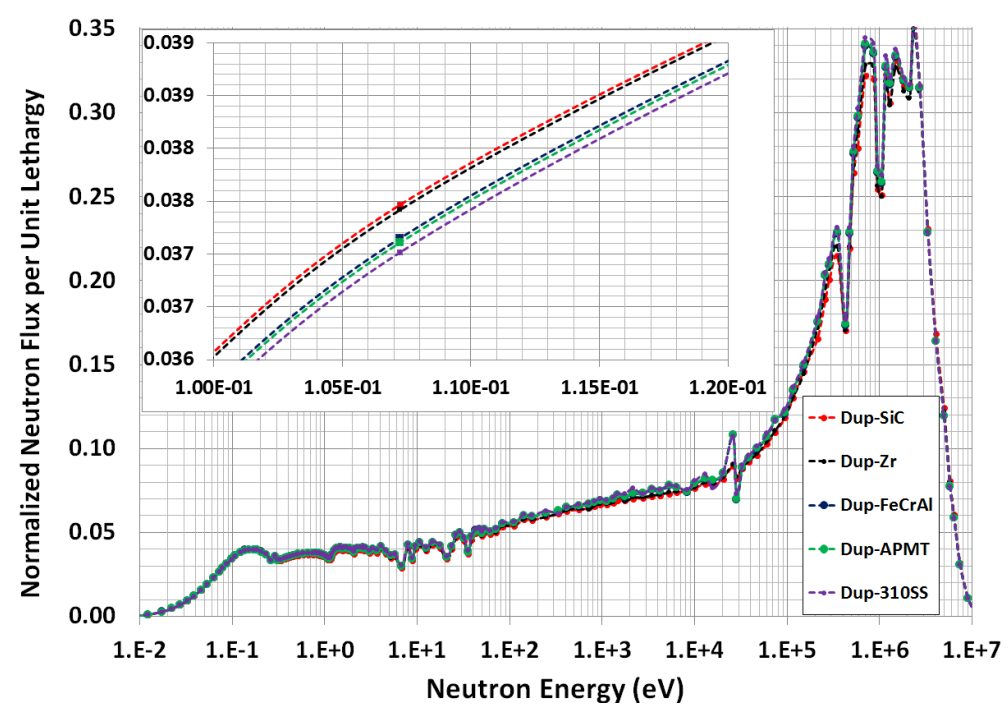

(b)

Fig. 8. Neutron spectra normalized per unit lethargy for candidate claddings at BOL: (a) $\mathrm{UO}_{2}$ fuel; (b) Duplex fuel.

Since $\mathrm{SiC}$ contains the least absorbing material among the candidate claddings, the highest inventory of thermal neutrons results, and candidate fuels with $\mathrm{SiC}$ cladding therefore exhibit a softer spectrum than with other claddings. In contrast, since FeCrAl, APMT and 310SS contain more absorbing materials, more thermal neutrons are absorbed in these claddings, resulting in an increase in the fast neutrons fraction in the system (Fig. 9), compared to the 
Zr and SiC cladding cases (George et al., 2015).

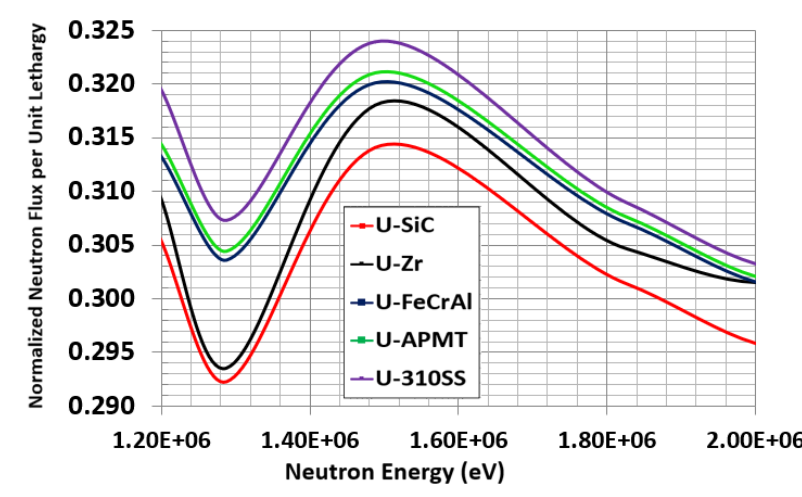

(a)

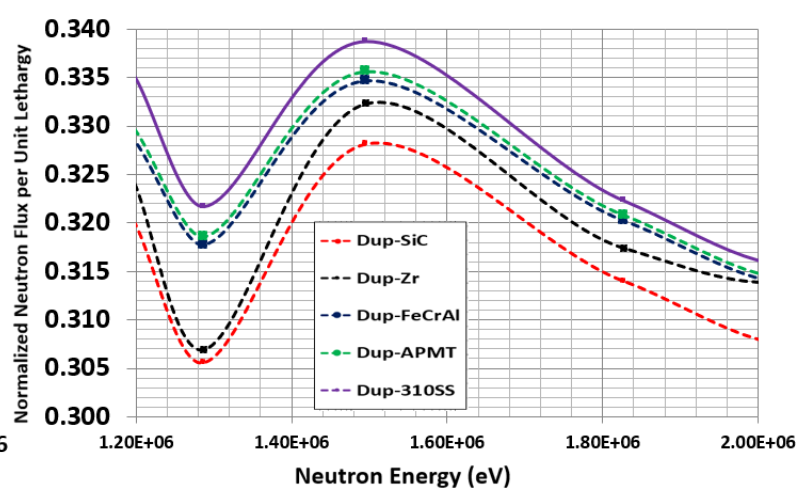

(b)

Fig. 9. Fast neutron spectra normalized per unit lethargy for candidate claddings at BOL: (a) $\mathrm{UO}_{2}$ fuel; (b) Duplex fuel.

Fig. 10 shows the normalized neutron flux ratio (the ratio of the neutron flux in the duplex fuel to that in the $\mathrm{UO}_{2}$ fuel) at BOL for all candidate claddings. It suggests that the duplex fuel yields a softer spectrum than the $\mathrm{UO}_{2}$ fuel in all cases; the ratio in the thermal range is between $\sim 1.05$ and $\sim 1.1$ (Franceschini and Petrović, 2008). This spectrum softening improves neutron economy and thus discharge burnup. Fig. 10 also exhibits the absorption resonance between $\sim 10$ to $\sim 100 \mathrm{eV}$ in the epithermal energy range, which is similar to the resonance structures observed for the normalized total cladding capture ratio (as shown in Fig. 7).

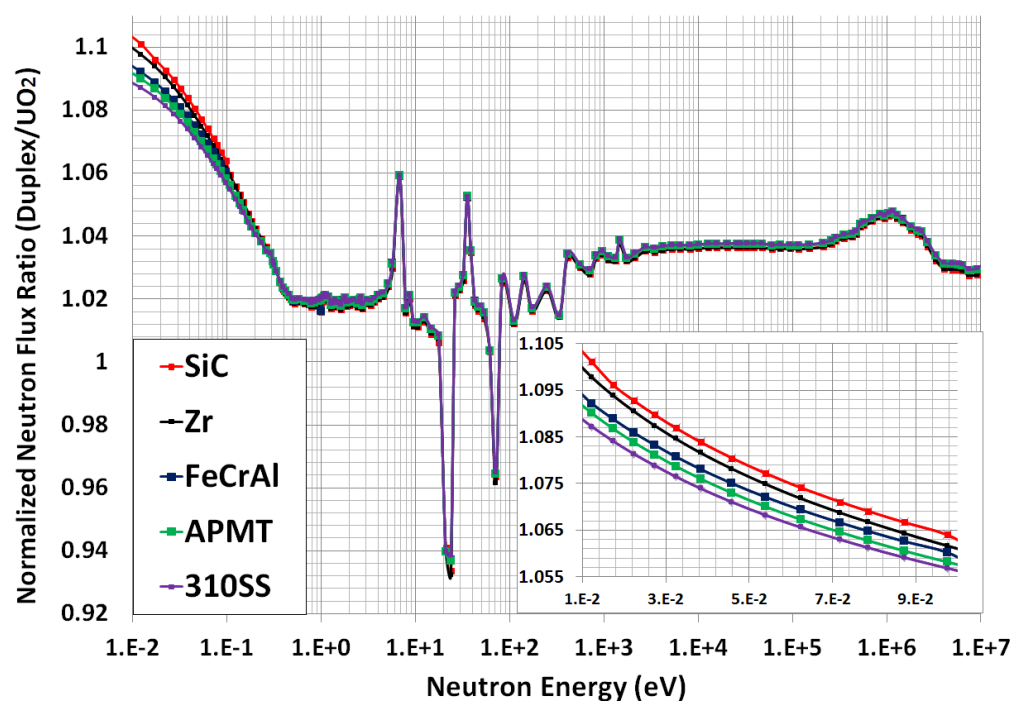

Fig. 10. Normalized neutron flux ratio (duplex: $\left.\mathrm{UO}_{2}\right)$ at BOL.

Fig. 11 shows the deviation from the Zr reference case in the normalized neutron spectra for all candidate claddings at BOL for both fuels. It can be seen in Figs. 11a and 11b that, 


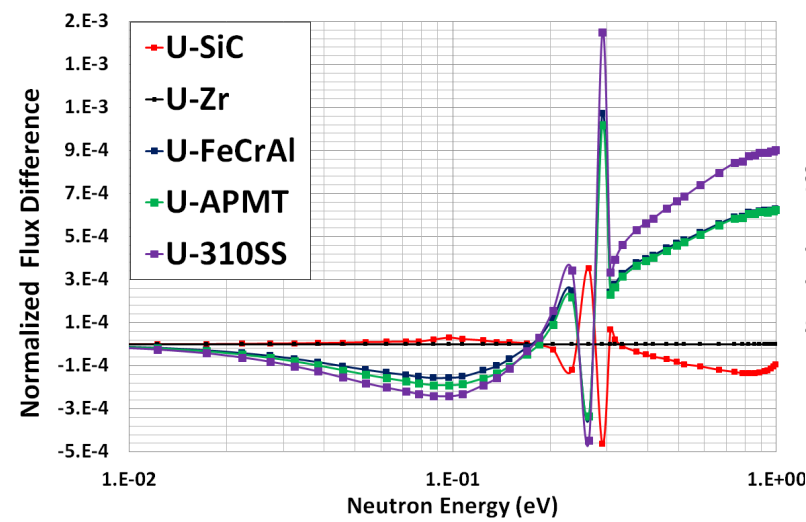

(a)

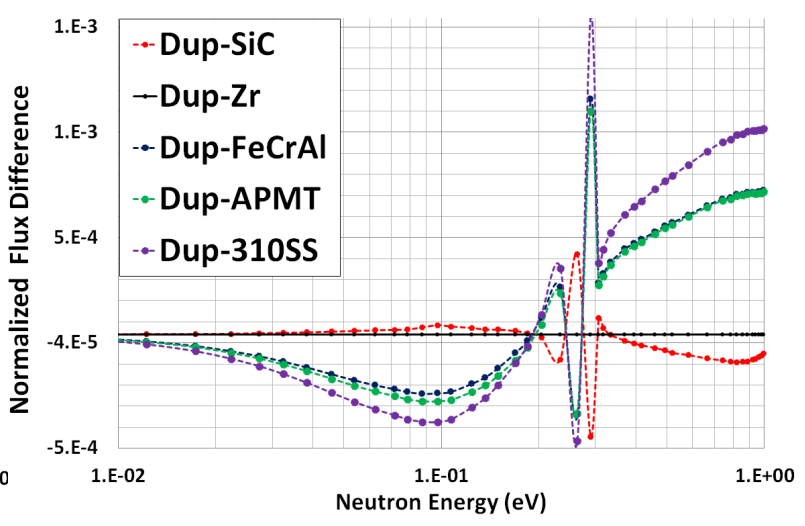

(b)

Fig. 11. Normalized neutron spectra deviation from reference $\mathrm{Zr}$ case at BOL: (a) $\mathrm{UO}_{2}$ fuel; (b) Duplex fuel.

with the exception of $\mathrm{SiC}$, the candidate ATF claddings have a slightly harder spectrum, due to enhanced thermal absorption. The lower neutron capture cross-section of $\mathrm{SiC}$ results in a softer spectrum compared to Zr.

\section{Analysis of the rim effect and plutonium build-up}

This section examines the impact of increased self-shielding on the burnup distribution and build-up of plutonium over the pellet radius for the candidate claddings. For this purpose, the fuel pellets are divided into 6 concentric rings of equal volume, as shown in Fig. 12, in order to analyse the radial power profile. In the case of the duplex fuel, 1 ring of $\mathrm{ThO}_{2}$ and the other five rings $\mathrm{UO}_{2}$ are considered. The fuel pin depletes largely from the outside inwards, and there's a strong flux gradient around the pin. The division into rings of equal volume enables edge effects due to self-shielding in the pellet to be observed (Alam, 2018, Brown et al., 2015).

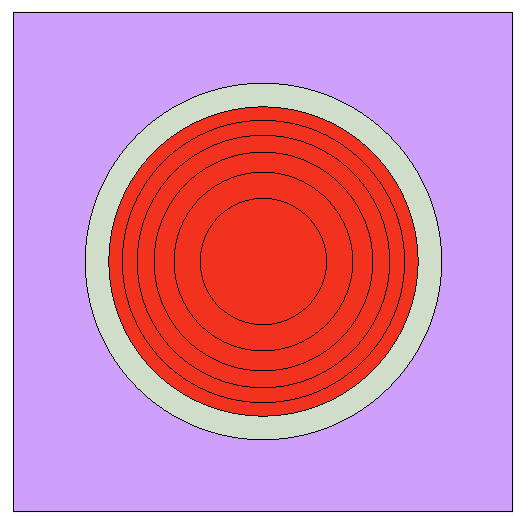

Fig. 12. Radial pin cell geometry with 6 equal-volume concentric rings. 
Figs. 13 and 14 show the relative fission power (RFP) across the ful pellet at BOL and end-of-life (EOL) for all candidate claddings for $\mathrm{UO}_{2}$ and duplex fuels, respectively. For the $\mathrm{UO}_{2}$ fuel, Fig. 13 shows that the RFP is largest in the region next to the cladding and smallest in the fuel centre, due to spatial self-shielding. The fission power distribution through the fuel pellet at BOL (Fig. 13a) is similar for all claddings, despite the differences in neutron capture cross-sections in the claddings. Due to spatial self-shielding, more ${ }^{239} \mathrm{Pu}$ is produced on the periphery of the fuel pellet than in its centre as the depletion cycle continues (Alam, 2018, George et al., 2015, Wu et al., 2015). The accumulation of fissile ${ }^{239} \mathrm{Pu}$ leads to an increase in RFP in the region next to the cladding, as shown in Fig. 13b. Fig. 13 also shows that the claddings with lower capture cross-sections ( $\mathrm{SiC}$ and $\mathrm{Zr}$ ) exhibit higher $\mathrm{RFP}$ in the outer region of the pellet. This is due to more thermal neutrons from the moderator passing through the cladding and causing fission in the fissile ${ }^{239} \mathrm{Pu}$ in the outer regions of the pellet (George et al., 2015).

In contrast, Fig. 14 shows that duplex fuel yields the highest RFP at the $\mathrm{UO}_{2}-\mathrm{ThO}_{2}$ interface and the lowest RFP in the region next to the cladding. During irradiation, the inner $\mathrm{UO}_{2}$ region is gradually depleted in ${ }^{235} \mathrm{U}$, while ${ }^{233} \mathrm{U}$ is bred in the outer $\mathrm{ThO}_{2}$ region. Thus, the RFP values peak at the $\mathrm{UO}_{2}-\mathrm{ThO}_{2}$ interface (Alam, 2018, Shwageraus et al., 2004). In addition, due to the absence of fissile material at BOL (Fig. 14a) in the outermost (100\% $\mathrm{ThO}_{2}$ ) region of the duplex fuel, RFP is lowest in the region next to the cladding. Things are different at EOL (Fig. 14b), where peripheral power is greater due to the breeding of ${ }^{233} \mathrm{U}$ over time.

Both candidate fuels exhibit similar fission power behaviour for all the candidate claddings, although there are differences in the amounts of fission power between the fuels.

The above phenomena can be explained by the rim effect in terms of middle-of-life (MOL) and EOL ${ }^{239} \mathrm{Pu}$ inventory as a function of pellet radial cross-section. ${ }^{239} \mathrm{Pu}$ has been chosen for this analysis since it is the largest contributor to fission after ${ }^{235} \mathrm{U}$, and its breeding significantly contributes to the core lifetime. For both fuels, Fig. 15 shows the ${ }^{239} \mathrm{Pu}$ accumulation in the fuel pellets with burnup. The ${ }^{239} \mathrm{Pu}$ inventory increases differently (bottom right corner of Fig. 15) with burnup for different claddings. As expected, the build-up of ${ }^{239} \mathrm{Pu}$ is higher in the $\mathrm{UO}_{2}$ fuel than in the duplex fuel.

For the $\mathrm{UO}_{2}$ fuel, the local build-up of ${ }^{239} \mathrm{Pu}$ on the periphery of the fuel pellet is significantly greater than in the centre due to strong epithermal ${ }^{238} \mathrm{U}$ resonance absorption, known as the rim effect (Alam, 2018, Brown et al., 2015) (Fig. 16a). ${ }^{239} \mathrm{Pu}$ accumulates with increasing burnup due to the spatial self-shielding of neutrons, whereby thermalized neutrons are captured in the outer region and thus shielded from the centre of the fuel. The higher $\mathrm{Pu}$ breeding at the pellet periphery results in very high burnup in the rim region and greater local fission gas release, which is responsible for the formation of porosity in this region. For the duplex fuel, the local build-up of ${ }^{233} \mathrm{U}$ (Fig. 17) on the periphery of the fuel pellet is significantly higher than that of ${ }^{239} \mathrm{Pu}$ (Fig. 16b).

Figs. $16 \mathrm{a}$ and $16 \mathrm{~b}$ show that the EOL ${ }^{239} \mathrm{Pu}$ inventories are higher for the claddings (Fe-based: FeCrAl, APMT and steel-based: 310SS) with higher thermal capture cross-sections due to spectral hardening, unlike $\mathrm{SiC}$ and $\mathrm{Zr}$. For both fuels, the Fe- and steel-based claddings absorb more thermal neutrons than $\mathrm{Zr}$ or SiC cladding, thereby increasing the fast neutron 


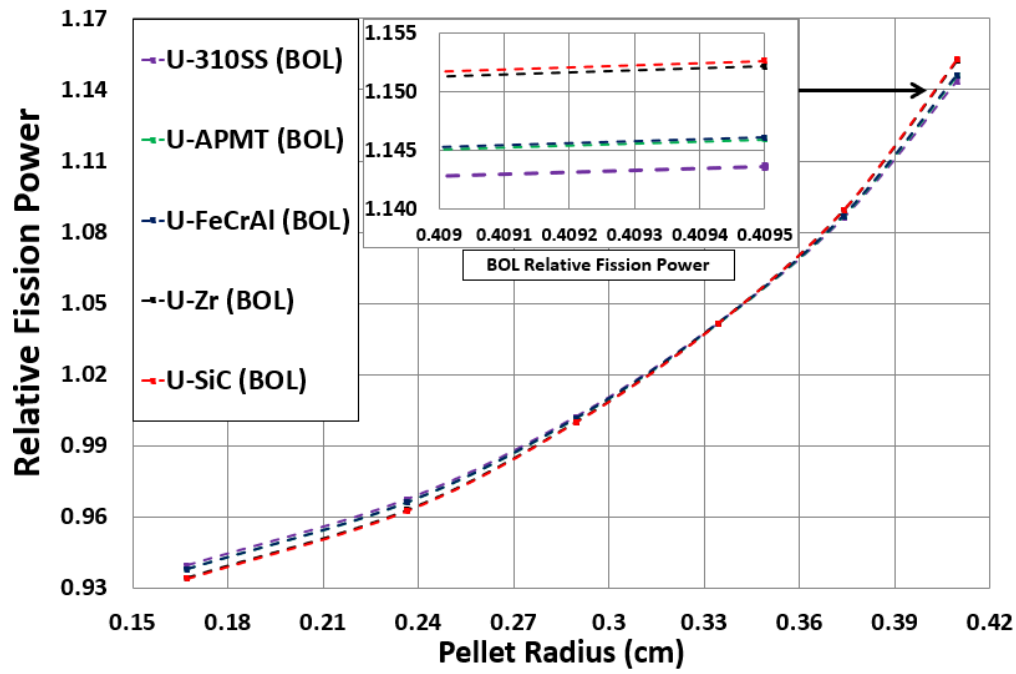

(a)

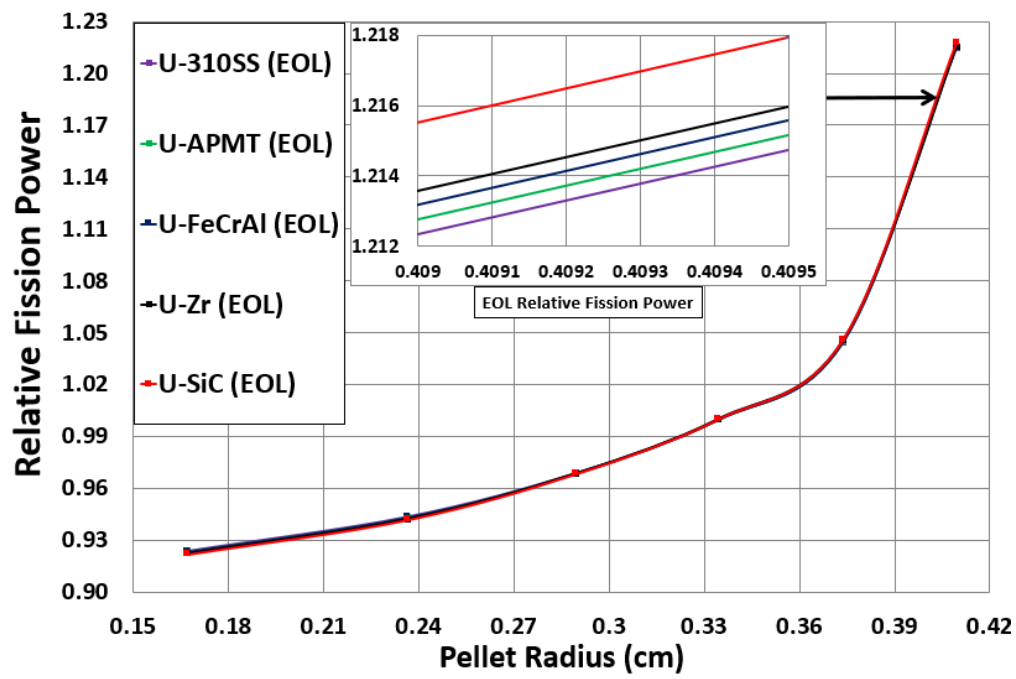

(b)

Fig. 13. Relative radial fission power distributions for $\mathrm{UO}_{2}$ fuel: (a) BOL (dashed lines); (b) EOL (solid lines). 


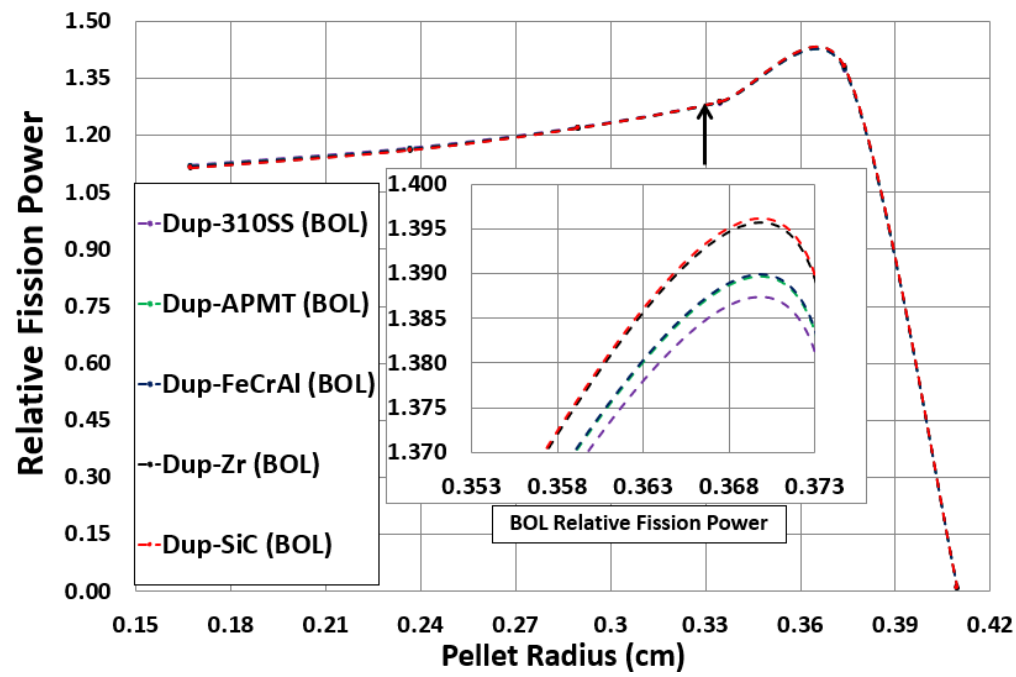

(a)

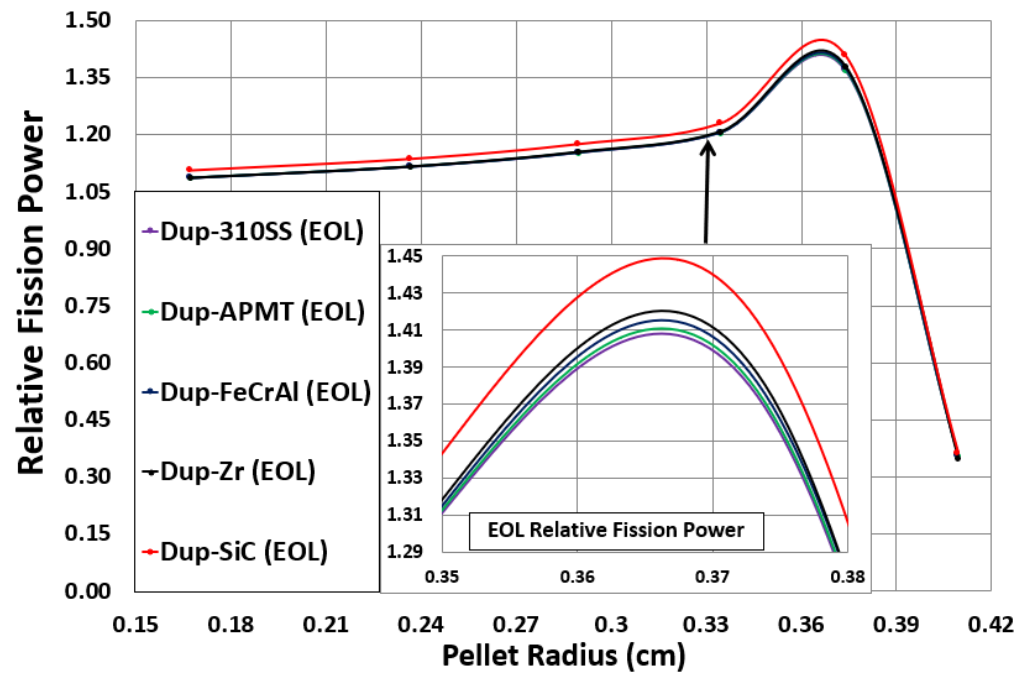

(b)

Fig. 14. Relative radial fission power distributions for duplex fuel: (a) BOL (dashed lines); (b) EOL (solid lines). 


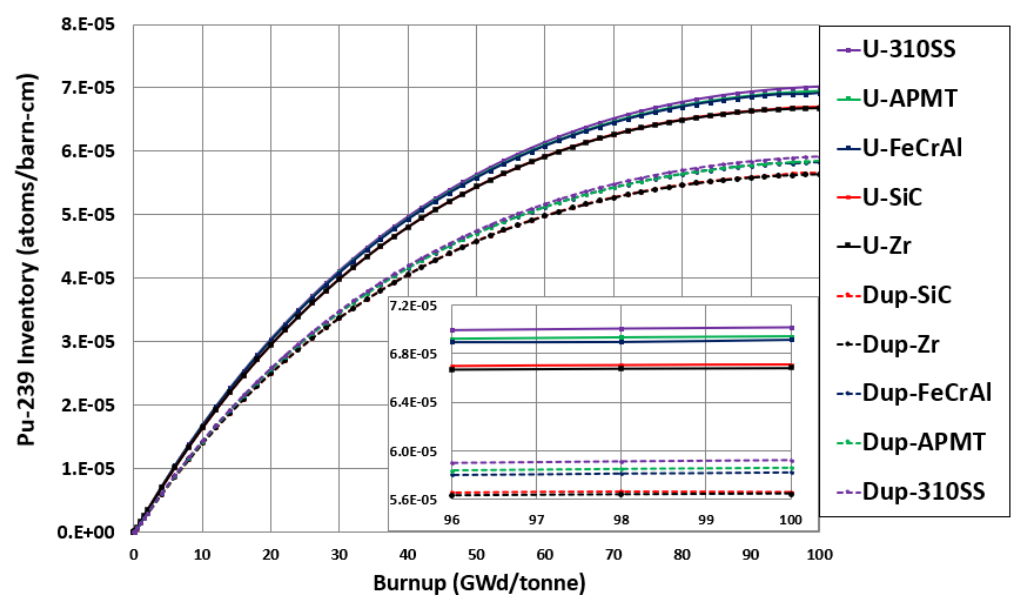

Fig. 15. ${ }^{239} \mathrm{Pu}$ inventory vs. burnup for both fuels and all candidate claddings.

fraction in the former. In turn, for $\mathrm{UO}_{2}$ fuel, this increases resonance capture in ${ }^{238} \mathrm{U}$, hence more $\mathrm{Pu}$ breeding is observed throughout the cycle for the Fe- and steel-based cladding fuels.

In contrast, for the duplex fuel (Fig. 16b), although significant ${ }^{239} \mathrm{Pu}$ breeding is observed in the $\mathrm{UO}_{2}$ region (radius of $3.9 \mathrm{~mm}$ ), a sharp decline in ${ }^{239} \mathrm{Pu}$ concentration is evident on the periphery of the pellet (radius of $4.095 \mathrm{~mm}$ ) due to the absence of ${ }^{238} \mathrm{U}$. Conversely, a sharp increase in ${ }^{233} \mathrm{U}$ is observed in the outermost region of duplex fuel (Fig. 17). Fig. 18 shows the ${ }^{233} \mathrm{U}$ accumulation in the duplex pellet with burnup, which varies (bottom right corner of Fig. 18) for different claddings. Since a duplex fuel pin is composed of a $\mathrm{UO}_{2}$ centre surrounded by an annulus of $\mathrm{ThO}_{2}$, the $\mathrm{ThO}_{2}$ region directly faces the thermal neutron flux incident from the moderator. The higher capture rate in the thorium results in efficient breeding of ${ }^{233} \mathrm{U}$. It can also be observed from Figs. 17 and 18 that, like ${ }^{239} \mathrm{Pu}$ (for $\mathrm{UO}_{2}$ fuel), the ${ }^{233} \mathrm{U}$ (for duplex fuel) inventories at EOL are higher for the claddings (Fe-based: FeCrAl, APMT and steel-based: 310SS) with higher thermal capture cross-sections, due to spectral hardening.

It can also be observed from Figs. 16a, 16b and 17 that EOL inventories for ${ }^{239} \mathrm{Pu}$ (for $\mathrm{UO}_{2}$ fuel) and ${ }^{233} \mathrm{U}$ (for duplex fuel) are higher for the Fe and steel-based claddings with higher thermal capture cross-sections. However, although $\mathrm{SiC}$ exhibits the lower capture cross-section than that of the $\mathrm{Zr}, \mathrm{SiC}$ provides higher ${ }^{239} \mathrm{Pu}$ (for $\mathrm{UO}_{2}$ fuel) and ${ }^{233} \mathrm{U}$ (for duplex fuel) inventories at EOL compared to Zr. There is a competition between the higher capture cross-section of $\mathrm{Zr}$ and the higher EOL RFP of SiC for the candidate fuels. Figs. 13b and 14b show for the candidate fuels that the EOL RFP values for the SiC claddings are significantly higher than Zr, causing higher thermal neutrons from the moderator passing through the $\mathrm{SiC}$ cladding (than that of the $\mathrm{Zr}$ cladding) and higher resonance capture in fertile ${ }^{238} \mathrm{U}$ (for $\mathrm{UO}_{2}$ fuel) and ${ }^{232} \mathrm{Th}$ (for duplex fuel). In fact, higher EOL RFP of SiC outperforms the higher capture cross-section of $\mathrm{Zr}$ and this results in a higher ${ }^{239} \mathrm{Pu}$ and ${ }^{233} \mathrm{U}$ breeding in $\mathrm{SiC}$ than that of the $\mathrm{Zr}$ cladding. 


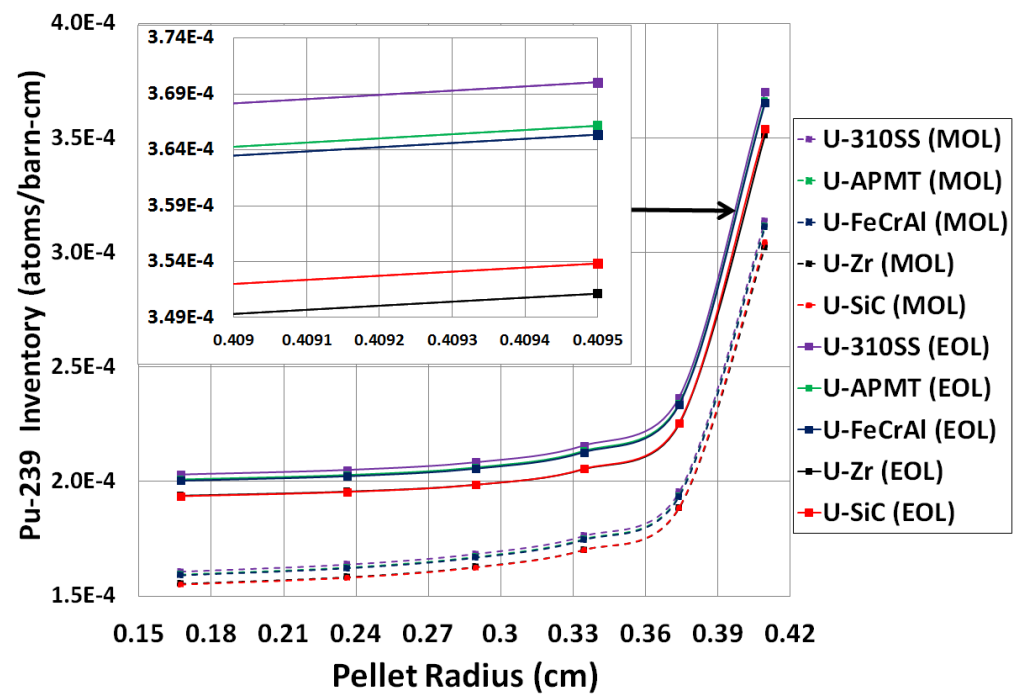

(a)

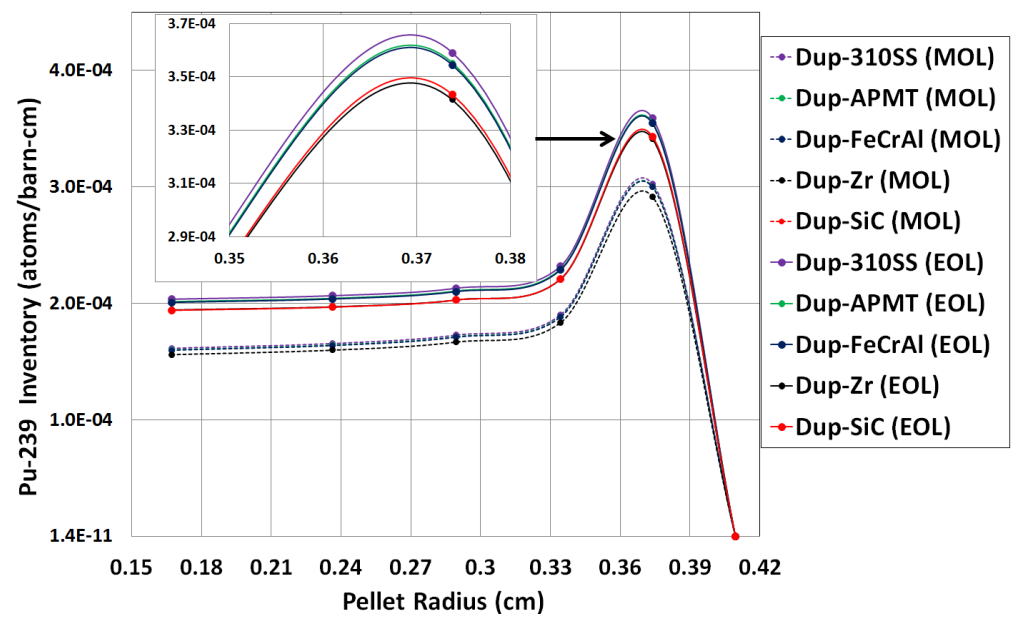

(b)

Fig. 16. Radial distributions of ${ }^{239} \mathrm{Pu}$ at MOL (dashed lines) and EOL (solid lines) for candidate claddings: (a) $\mathrm{UO}_{2}$ fuel; (b) Duplex fuel. 


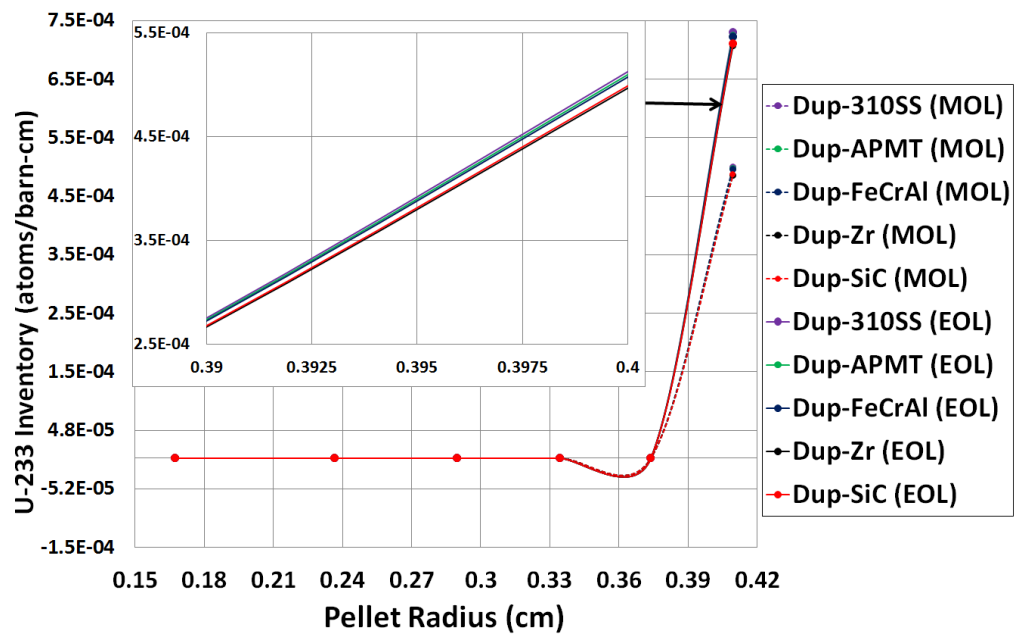

Fig. 17. Radial distributions of ${ }^{233} \mathrm{U}$ at MOL (dashed lines) and EOL (solid lines) for candidate claddings with duplex fuel.

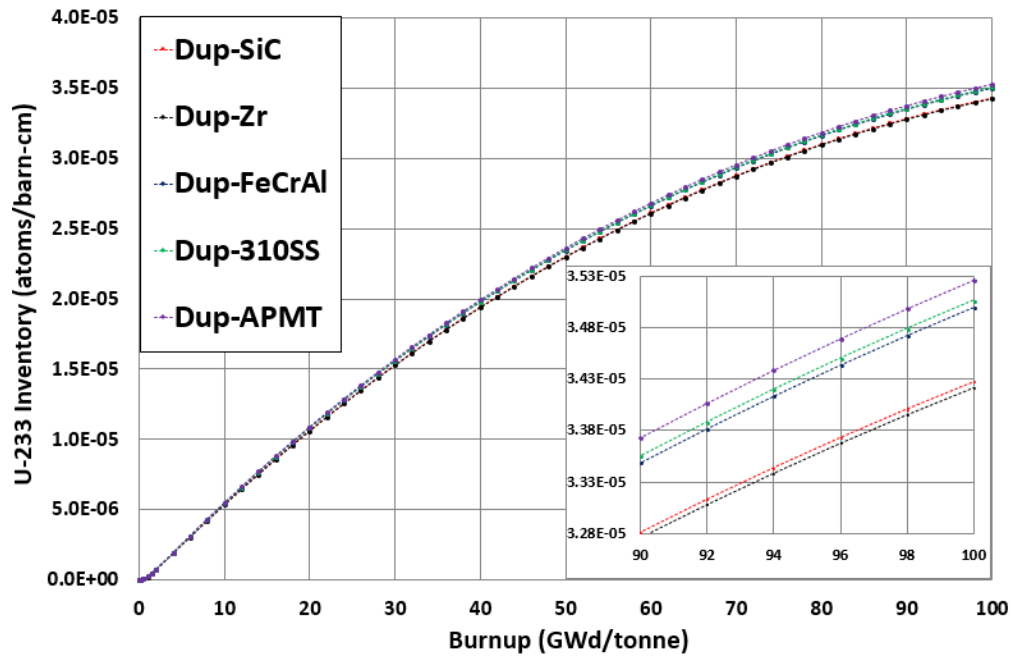

Fig. 18. ${ }^{233} \mathrm{U}$ inventory vs. burnup for candidate claddings with duplex fuel. 


\section{Reactivity feedback analysis}

It is important to evaluate the impact of the candidate claddings on reactivity coefficients in order to determine whether they provide similar feedback responses as for the reference $\mathrm{Zr}$ cladding. In keeping with the safety standards of operational PWRs, it is necessary that each fuel-cladding combination maintains negative reactivity coefficients. In this section, assembly-level reactivity coefficients - moderator temperature coefficient (MTC) and fuel temperature coefficient (FTC) - are evaluated at hot full power conditions. As observed in previous studies (Alam, 2018), for both fuels, reactivity coefficient values are higher (less negative) at BOL and decrease (become more negative) with burnup, due to the changes in isotopic composition and the increased variety of isotopes present. MTC and FTC values are evaluated only at BOL since this is the most limiting state.

FTC values are evaluated by increasing the fuel temperature by $20 \mathrm{~K}$ from the reference value of $900 \mathrm{~K}$. MTC values are calculated by increasing the coolant temperature by $20 \mathrm{~K}$ and modifying the water density accordingly.

Table 5 shows that the MTC values are strongly negative due to the absence of soluble boron. The MTC values are more negative for the 310SS, APMT and FeCrAl claddings than for $\mathrm{Zr}$ and $\mathrm{SiC}$. It can also be seen that MTC values are more negative in the duplex fuel for all claddings.

\begin{tabular}{|l|c|c|}
\hline Cladding & $\mathbf{U O}_{2}$ & Duplex \\
\hline Zircaloy & -24.31 & -28.24 \\
\hline $\mathrm{SiC}$ & -24.59 & -28.39 \\
\hline $\mathrm{FeCrAl}$ & -24.85 & -28.91 \\
\hline $\mathrm{APMT}$ & -25.01 & -29.20 \\
\hline $310 \mathrm{SS}$ & -25.85 & -30.05 \\
\hline
\end{tabular}

Table 5. BOL MTC $(\mathrm{pcm} / \mathrm{K})$ for a $20 \mathrm{~K}$ change in coolant temperature.

The differences in MTC for the candidate claddings are primarily due to differences in the resonance capture within the fuel and cladding (Brown et al., 2015). The resonant behaviour can be appreciated by observing the normalized total capture in fuel and cladding per unit lethargy at BOL (Fig. 19). This is indicative of the extent to which resonance capture plays a role, and the resultant impact on MTC.

The plots for $\mathrm{UO}_{2}$ fuel (Fig. 19a) and duplex fuel (Fig. 19b) show that 310SS, APMT and $\mathrm{FeCrAl}$ exhibit resonant behaviour in the range 10-100 eV and thereby provide lower (more negative) MTC values compared to the $\mathrm{Zr}$ and $\mathrm{SiC}$ cases. Normalized total capture rates in fuel and cladding are shown at $\sim 20 \mathrm{eV}$ in the top right corners of Figs. 19a and 19b to shed light on this resonant behaviour. The MTC values are most negative for the 310SS and APMT claddings due to the presence of Mo and Ni, and Mo isotopes only, respectively. Fig. 5b shows the capture cross-sections of different Mo isotopes (Brown et al., 2015). It can be seen that Mo is a very strong resonance absorber, which impacts the MTC and shutdown margin significantly (Brown et al., 2015). These strong resonances illustrate why Mo-containing claddings (APMT and 310SS) are unattractive for a long-life marine core. 


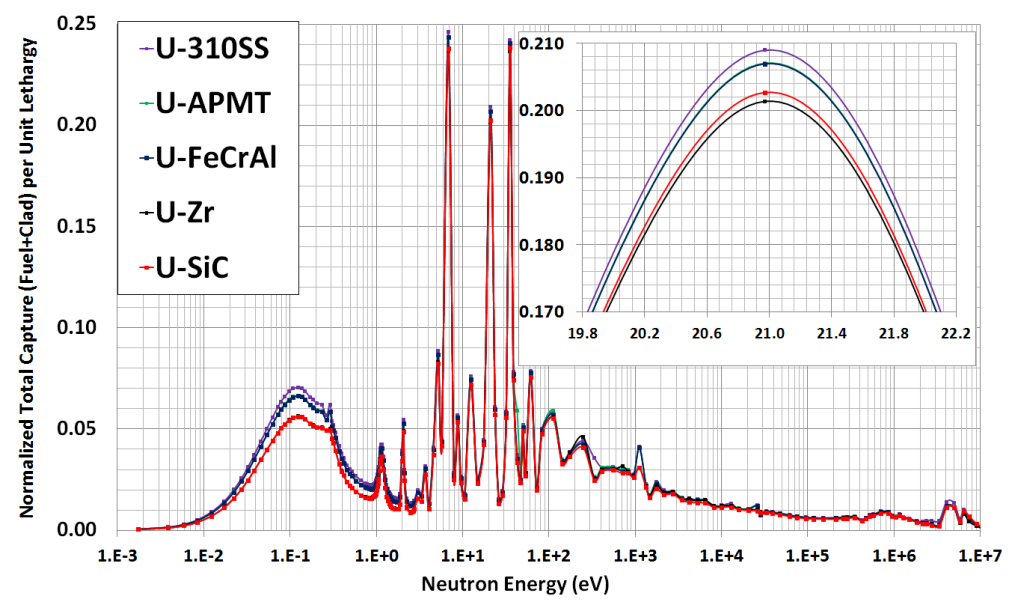

(a)

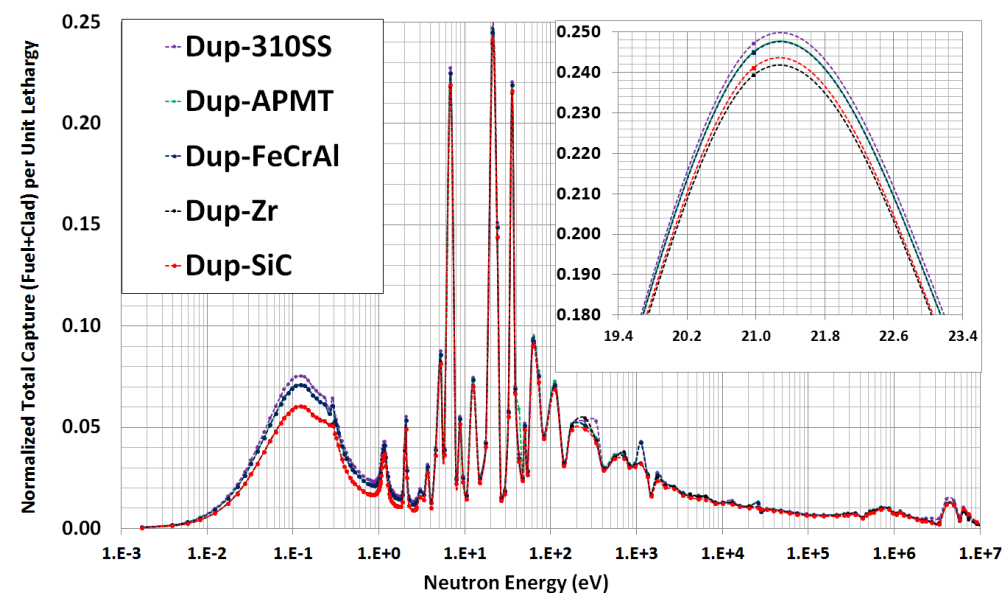

(b)

Fig. 19. Normalized total capture rates in the fuel and cladding per unit lethargy at BOL: (a) $\mathrm{UO}_{2}$ fuel; (b) Duplex fuel. 
Resonance absorption in Mo cannot be mitigated entirely by isotopic enrichment and/or reducing cladding thickness.

Fig. 20 shows the difference in normalized total capture rates in fuel and cladding between the duplex and $\mathrm{UO}_{2}$ fuels with the candidate claddings at BOL. Claddings with higher capture cross-sections (310SS, APMT and FeCrAl) exhibit larger differences. Table 5 shows that the differences in MTC values for the $\mathrm{UO}_{2}$ and duplex fuels are larger for the claddings with higher capture cross-sections ( $\sim 17 \%$ difference) than those with lower capture cross-sections ( $\sim 15 \%$ difference).

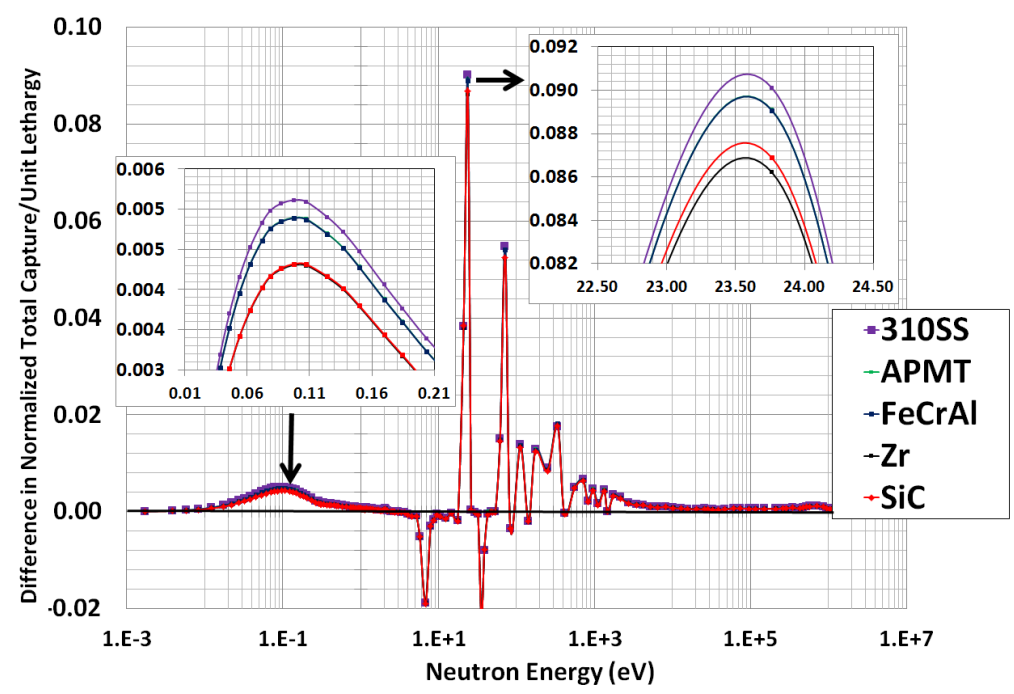

Fig. 20. Differences in normalized total capture rates in fuel and cladding between duplex and $\mathrm{UO}_{2}$ fuels with candidate claddings at BOL.

From analysis in previous studies (Alam, 2018, Alam et al., 2016), it is known that capture in plutonium also affects the MTC. Fig. 21 presents the differences in ${ }^{239} \mathrm{Pu}$ capture between the duplex and $\mathrm{UO}_{2}$ fuels with candidate claddings at a burnup of $0.20 \mathrm{GWd} /$ tonne, showing the duplex fuel experiences more ${ }^{239} \mathrm{Pu}$ captures than the $\mathrm{UO}_{2}$ fuel. There are larger differences between the ${ }^{239} \mathrm{Pu}$ capture rates for the two fuels for claddings with higher capture cross-sections (310SS and APMT), consistent with the results obtained for total capture (Fig. 20). Due to the higher total capture rates associated with higher ${ }^{239} \mathrm{Pu}$ capture, fuelcladding combinations with harder spectra thus have more negative MTC than combinations with softer spectra.

Table 6 shows that the BOL FTC values are negative for all fuel-cladding combinations. The minimal variation in FTC observed for a given candidate fuel with different claddings is due to the identical ${ }^{235} \mathrm{U}$ enrichment and cladding thickness (George et al., 2015). It is therefore expected that each fuel-cladding combination will maintain similar operational standards as regards FTC as for Zr cladding.

For both fuels, the 310SS and APMT claddings exhibit marginally more negative FTC values. This can be attributed to the differences in the Doppler broadening of total fertile absorption. Figs. 22a and 22b show the deviation in fertile capture rate per unit lethargy at 


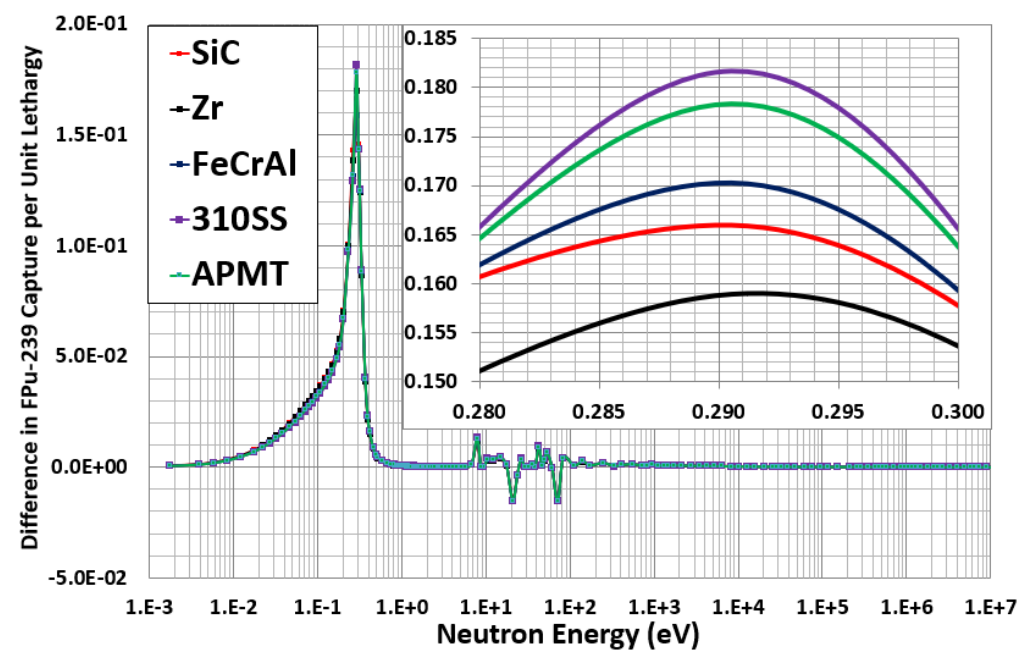

Fig. 21. Differences in ${ }^{239} \mathrm{Pu}$ capture rates between duplex and $\mathrm{UO}_{2}$ fuels with candidate claddings at 0.20 GWd/tonne.

\begin{tabular}{|l|c|c|}
\hline Cladding & $\mathbf{U O}_{2}$ & Duplex \\
\hline Zircaloy & -2.18 & -2.97 \\
\hline $\mathrm{SiC}$ & -2.19 & -2.98 \\
\hline FeCrAl & -2.21 & -3.01 \\
\hline APMT & -2.22 & -3.02 \\
\hline 310SS & -2.24 & -3.03 \\
\hline
\end{tabular}

Table 6. BOL FTC $(\mathrm{pcm} / \mathrm{K})$ for a $20 \mathrm{~K}$ change in fuel temperature. 


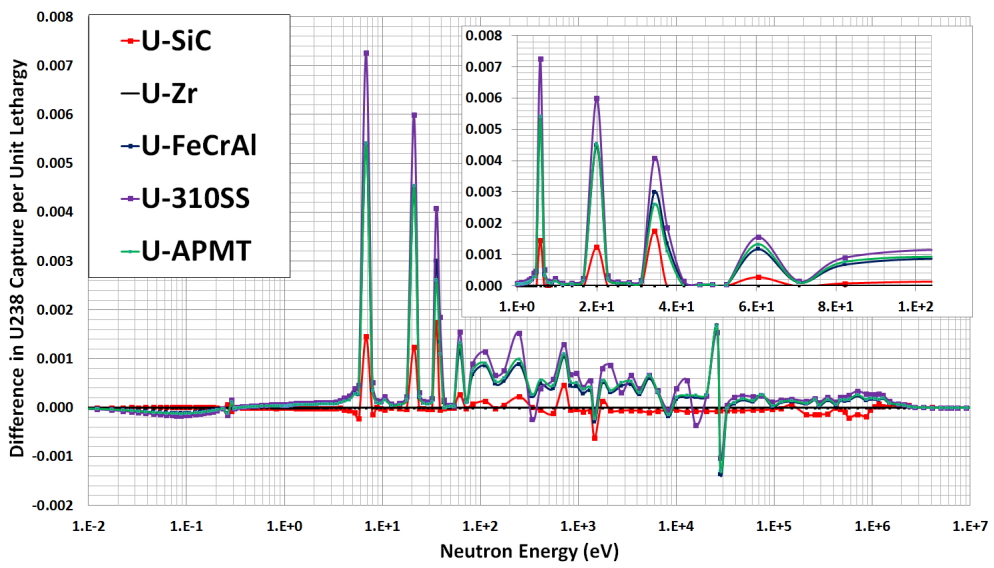

(a)

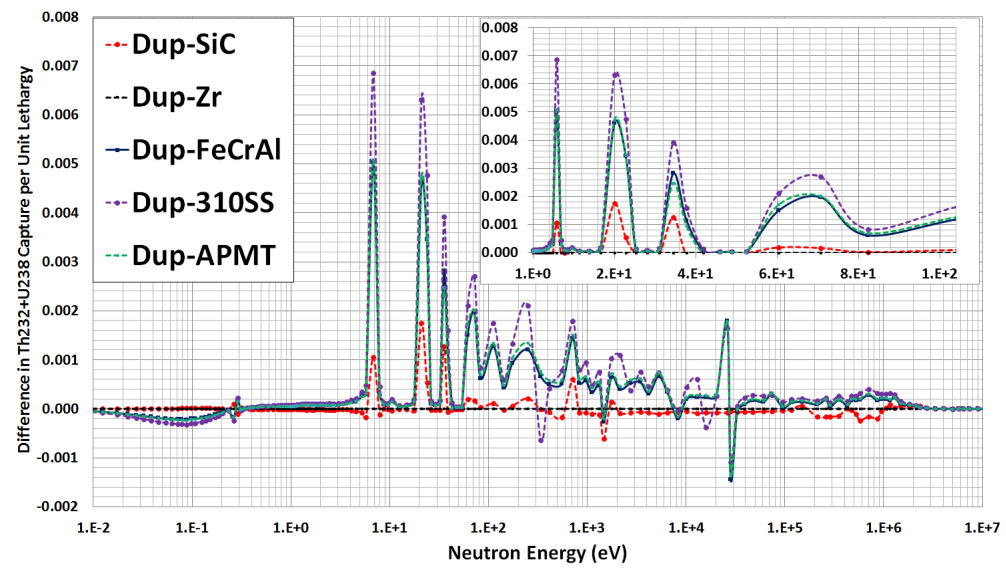

(b)

Fig. 22. Deviation in fertile capture rate per unit lethargy from reference $\mathrm{Zr}$ cladding case: (a) $\mathrm{UO}_{2}$ fuel; (b) Duplex fuel. 
BOL from the reference $\mathrm{Zr}$ cladding case for the different cladding options with the $\mathrm{UO}_{2}$ and duplex fuels, respectively. The larger fertile capture rates in the $6-35 \mathrm{eV}$ range for the $310 \mathrm{SS}$ and APMT claddings contribute to their more negative FTC values.

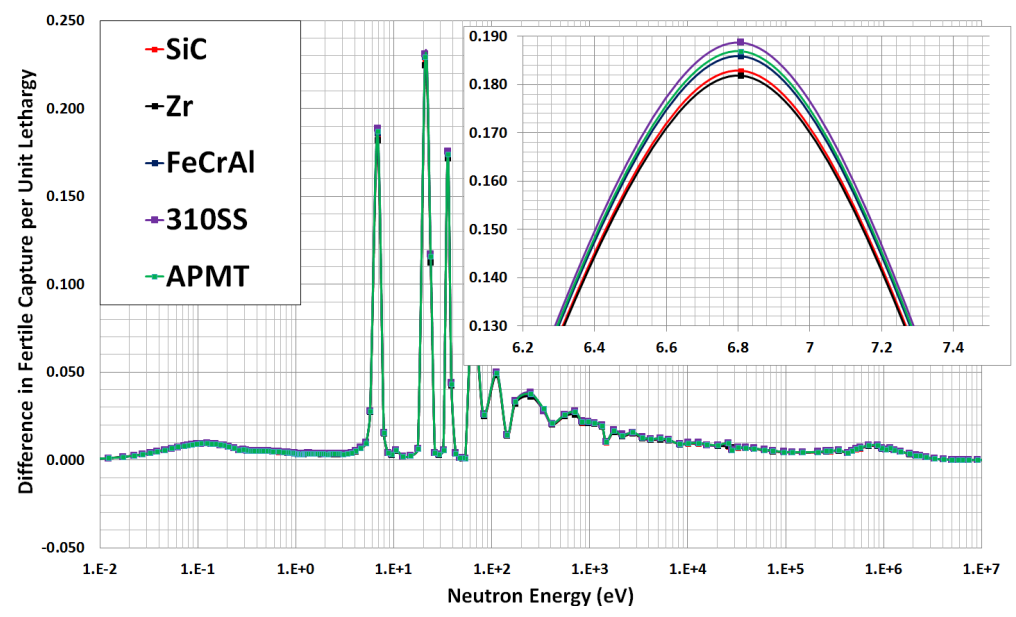

Fig. 23. Differences in total fertile capture rates between duplex and $\mathrm{UO}_{2}$ fuels with candidate claddings at BOL.

Fig. 23 shows the difference in total fertile capture rates in fuel and cladding per unit lethargy between duplex and $\mathrm{UO}_{2}$ fuel for the candidate claddings. Again, a larger difference is observed for the claddings with higher capture cross-sections (310SS and APMT). Consistent with the MTC findings, it can be concluded that fuel-cladding combinations with harder spectra provide more negative FTC due to the resulting higher rates of fertile capture.

Since reactivity feedback calculations do not account for the presence of leakage, it is important to address from the previous study (Alam, 2018) that reactivity feedback parameters (both MTC and FTC) are more negative in the whole-core environment and therefore, will not be a limiting case while considering these ATF claddings in the proposed SMR SBF whole-core.

\section{Practical considerations for the duplex fuel}

The major challenge for the micro-heterogeneous duplex fuel arrangements is to meet the thermal-hydraulic margins since the most severe situation will be in the duplex pellet case where most of the power is generated in the $\mathrm{UO}_{2}$ part of the fuel pellet (at least during the first few years of operation). This phenomenon leads to a very high local power peaking in the central $\mathrm{UO}_{2}$ region of the duplex pellet (Zhao, 2001, MacDonald and Lee, 2004). At BOL, the release of fission energy for the duplex fuel will only be in the inner $\mathrm{UO}_{2}$ pellet (region) leading to higher fuel centerline temperatures in the duplex fuel pellet than that of the $\mathrm{UO}_{2}$ fuel (Alam, 2018, Shwageraus et al., 2004). In order to confirm that all the thermal-hydraulic constraints are satisfied for the duplex fuel, 3D neutronic/thermal-hydraulic coupling of hybrid monte carlo MONK with sub-channel analysis COBRA-EN code for hot channel analysis (Alam, 2018) has been performed to evaluate key thermal-hydraulic parameters 
such as: minimum departure from nucleate boiling ratio (MDNBR), heat flux, cladding, inner surface and fuel centreline temperatures, and pressure drop. Our study confirmed that thermal-hydraulic design requirements for the duplex fuel can be met and there will be no melting in the $\mathrm{UO}_{2}$ region of the duplex pellet. Since thermal-hydraulic design requirements are met by a good margin, it can, therefore, be expected that other issues (e.g. hydriding of cladding, fission gas release, and pellet/cladding mechanical interactions) arising from the large temperature gradients in the $\mathrm{UO}_{2}$ part of the fuel pellet can be avoided. These issues are out of the scope of this paper and detailed hot channel thermal-hydraulic analysis can be found in the first author's PhD research (Alam, 2018).

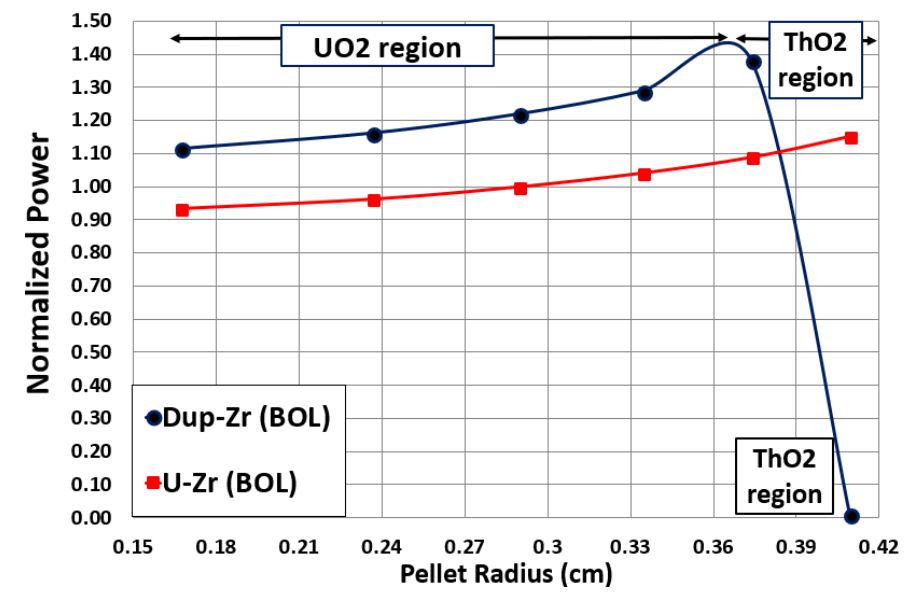

Fig. 24. Normalized power density distribution for duplex fuel (at the $\mathrm{UO}_{2}-\mathrm{ThO}_{2}$ interface) and all-UO fuel.

The power peaking problem is also observed for the duplex fuel for the $\mathrm{Zr}$ case plotted as a function of normalized power along the duplex pellet radius at BOL. Fig. 24 shows that normalized power of duplex pellet (at the $\mathrm{UO}_{2}-\mathrm{ThO}_{2}$ interface) is about a factor of 1.4 at the BOL and almost $20 \%$ higher than the all- $\mathrm{UO}_{2}$ fuel. It is clear that power peaking can be kept below standard industry limit of 1.5 (Pramuditya and Takahashi, 2013). It is worthwhile mentioning that previous studies (Alam, 2018, Shwageraus et al., 2004) exhibit a normalized power of 2.4 for axially micro-heterogeneous duplex fuel and this higher BOL normalized power $\mathrm{UO}_{2}$ region results in an unacceptably high fuel temperature. In order to avoid this issue, our radial micro-heterogeneous duplex fuel is designed in such a way that $\mathrm{ThO}_{2}$ region is $\sim 25 \%$ of the $\mathrm{UO}_{2}$ region and normalized power is limited to 1.4 , which is an obvious design improvement considering the practical perspective. A detailed discussion of the radial micro-heterogeneous duplex fuel design and analyses can be found in the first author's PhD research (Alam, 2018).

Fuel performance and fabrication issues for the duplex fuel are worth investigating in the future. It is also worth addressing that the main objective of this paper is to evaluate the neutronic feasibility of the duplex fuel with the candidate claddings in a SBF environment. We have considered the issues of peak temperatures, local burnup, fuel performance issues of duplex fuel in the previous study (Alam, 2018) and these issues are, however, out of the 
scope of this paper.

\section{Conclusions}

An assembly-level reactor physics assessment examining reactivity, achievable discharge burnup, spectral variations, rim effect and reactivity feedback parameters associated with a number of candidate ATF claddings was undertaken in comparison to reference Zircaloy cladding for a long-life marine PWR core. The key findings are:

- The lower neutron capture and relatively higher discharge burnup for $\mathrm{SiC}$ make it the best candidate cladding for the target application.

- Cladding capture is higher in duplex fuel than $\mathrm{UO}_{2}$ fuel for all of the ATF claddings. In addition, duplex fuel contributes to $\sim 5-10 \%$ and $\sim 2-3 \%$ higher cladding capture than $\mathrm{UO}_{2}$ fuel with the candidate claddings in the thermal and fast energy ranges, respectively.

- Claddings with lower capture cross-sections ( $\mathrm{SiC}$ and $\mathrm{Zr}$ ) exhibit higher relative fission power in the outer region of the fuel pellet.

- Unsurprisingly, the build-up of ${ }^{239} \mathrm{Pu}$ is higher in the $\mathrm{UO}_{2}$ fuel than in the duplex fuel for all the candidate claddings. EOL ${ }^{239} \mathrm{Pu}$ (for $\mathrm{UO}_{2}$ fuel) and ${ }^{233} \mathrm{U}$ (for duplex fuel) inventories are higher for the Fe-based: FeCrAl, APMT and steel-based: 310SS claddings with higher thermal capture cross-sections due to spectral hardening. However, there is an exception for $\mathrm{SiC}$ and $\mathrm{Zr}$ claddings. Although $\mathrm{SiC}$ exhibits the lower capture cross-section than that of the $\mathrm{Zr}, \mathrm{SiC}$ provides higher ${ }^{239} \mathrm{Pu}$ (for $\mathrm{UO}_{2}$ fuel) and ${ }^{233} \mathrm{U}$ (for duplex fuel) inventories at EOL compared to Zr. This is due to the fact that SiC's significantly higher EOL RFP outperforms the higher capture cross-section of Zr.

- Almost identical reactivity feedback parameters (MTC and FTC) were found for the $\mathrm{Zr}, \mathrm{SiC}$ and FeCrAl claddings; the Mo-containing claddings (APMT and 310SS) exhibit more negative MTC than the reference cladding, which is detrimental to shutdown margin. The fuel-cladding combinations with harder spectra exhibit more negative MTC and FTC values than those with softer spectra. MTC and FTC values are also observed to be more negative in the duplex fuel than the $\mathrm{UO}_{2}$ fuel for all the candidate claddings.

- The duplex fuel yields a softer spectrum than the $\mathrm{UO}_{2}$ fuel with the candidate claddings, thereby improving neutron economy and thus discharge burnup. For all cladding options considered, $\mathrm{UO}_{2}$ fuel will require higher burnable poison loadings than duplex fuel due to its higher initial excess reactivity.

It can be recommended from this study that with $\mathrm{SiC}$ a standard cladding thickness could be used with marginally less enriched uranium fuel. In contrast, to overcome the reactivity penalty associated with using an Fe- and Mo-based steel alloy cladding (FeCrAl, 310SS and 
APMT), the cladding thickness should be reduced and the fuel enrichment slightly increased, if the cycle length achieved with Zr cladding is to be matched.

Future work will focus on the optimization of the fuel enrichment and cladding thickness of Fe- and Mo-based steel alloy claddings in order to match the discharge burnup of the reference Zr case. These ATF fuel cladding concepts will also be evaluated for the high-power-density marine whole-core environment. Finally, an economic assessment will be undertaken to evaluate the fuel assembly cost when utilizing the candidate ATF cladding materials.

\section{References}

Alam, S.B., 2018. The Design of Reactor Cores for Civil Nuclear Marine Propulsion. Ph.D. thesis. University of Cambridge. Cambridge, UK.

Alam, S.B., Lindley, B.A., Parks, G.T., Shwageraus, E., 2016. Burnable poison designs for a soluble-boron-free civil nuclear marine PWR core, in: Proc. PHYSOR 2016, Sun Valley, ID. pp. 1926-1938.

Alam, S.B., Ridwan, T., Parks, G.T., Almutairi, B., Goodwin, C.S., 2018a. High power density reactor core design for civil nuclear marine propulsion. Part I: Assembly-level analysis, in: Proc. PHYSOR 2018, Cancun, Mexico. pp. 46-57.

Alam, S.B., Ridwan, T., Parks, G.T., Almutairi, B., Goodwin, C.S., 2018b. High power density reactor core design for civil nuclear marine propulsion. Part II: Whole-core analysis, in: Proc. PHYSOR 2018, Cancun, Mexico. pp. 58-69.

Andrews, N., Pilat, E., Shirvan, K., Kazimi, M.S., 2014. Impact of SiC cladding on plutonium burning in a thorium fueled PWR, in: Proc. ICAPP 2014, Charlotte, USA.

Aspelund, A., Molnvik, M., De Koeijer, G., 2006. Ship transport of $\mathrm{CO}_{2}$ : technical solutions and analysis of costs, energy utilization, energy efficiency and $\mathrm{CO}_{2}$ emissions. Chem. Eng. Res. Des. 84, 847-855.

Azevedo, C.R.F., 2011. Selection of fuel cladding material for nuclear fission reactors. Eng. Fail. Anal. 18, 1943-1962.

Brown, N.R., Todosow, M., Cuadra, A., 2015. Screening of advanced cladding materials and UN-U $\mathrm{Si}_{5}$ fuel. J. Nucl. Mater. 462, 26-42.

Bukharin, O., 2006. Russia's nuclear icebreaker fleet. Sci. Global Secur. 14, 25-31.

Carlton, J., Smart, R., Jenkins, V., 2011. The nuclear propulsion of merchant ships: aspects of engineering, science and technology. J. Mar. Eng. Technol. 10, 47-59.

Clayton, J., 1993. The Shippingport Pressurized Water Reactor and Light Water Breeder Reactor. Technical Report WAPD-T-3007. Westinghouse Electric Corporation, Bettis Atomic Power Laboratory. West Mifflin, PA.

Dedes, E., Turnock, S., Hudson, D., Hirdaris, S., 2011. Possible power train concepts for nuclear powered merchant ships, in: Proc. Int. Conf. Technologies, Logistics and Modelling for Low Carbon Shipping, Glasgow, UK. pp. 261-274.

Franceschini, F., Petrović, B., 2008. Core physics analysis of 100\% MOX core in IRIS. Ann. Nucl. Energy 35 (9), 1587-1597.

Galperin, A., Shwageraus, E., Todosow, M., 2002. Assessment of homogeneous thorium/uranium fuel for Pressurized Water Reactors. Nucl. Technol. 138, 111-122.

George, N.M., 2015. Assessment of Reactivity Equivalence for Enhanced Accident Tolerant Fuels in Light Water Reactors. Ph.D. thesis. University of Tennessee-Knoxville. Knoxville, TN.

George, N.M., Terrani, K.A., Powers, J., Worrall, A., Maldonado, I., 2015. Neutronic analysis of candidate accident-tolerant cladding concepts in PWR. Ann. Nucl. Energy 75, 703-712.

Hirdaris, S., Cheng, Y., Shallcross, P., Bonafoux, J., Carlson, D., Prince, B., Sarris, G., 2014. Considerations on the potential use of nuclear small modular reactor (SMR) technology for merchant marine propulsion. Ocean Eng. 79, 101-130.

Kazimi, M., Czerwinski, K., Driscoll, M., Hejzlar, P., Meyer, J., 1999. On the Use of Thorium in Light 
Water Reactors. Technical Report MIT-NFCTR-016. Department of Nuclear Engineering, Massachusetts Institute of Technology. Cambridge, MA.

Khlopkin, N., Zotov, A., 1997. Merchant marine nuclear-powered vessels. Nucl. Eng. Des. 173, $201-205$.

Kramer, A., 1962. Nuclear Propulsion for Merchant Ships. US Atomic Energy Commission, Washington, DC.

Leppänen, J., Pusa, M., 2009. Burnup calculation capability in the PSG2/Serpent Monte Carlo reactor physics code, in: Proc. Int. Conf. Advances in Mathematics, Computational Methods, and Reactor Physics, Saratoga Springs, NY.

Lindley, B.A., Kotlyar, D., Parks, G.T., Lillington, J.N., Petrovic, B., 2016. Reactor physics modelling of accident tolerant fuel for LWRs using ANSWERS codes. EPJ Nuclear Sci. Technol. 2.

Long, D., Richards, S., Smith, P., Baker, C., Bird, A., Davies, N., Dobson, G., Fry, T., Hanlon, D., Perry, R., Shepherd, M., 2015. MONK10: A Monte Carlo code for criticality analysis, in: Proc. Int. Conf. Nuclear Criticality Safety (ICNC 2015), Charlotte, NC. pp. 923-935.

MacDonald, P., Lee, C., 2004. Use of thoria-urania fuels in PWRs: A general review of a NERI project to assess feasible core designs, economics, fabrication methods, in-pile thermal/mechanical behavior, and waste form characteristics. Nucl. Technol. 147, 1-7.

Naceur, A., Marleau, G., 2018. Neutronic analysis for accident tolerant cladding candidates in CANDU-6 reactors. Ann. Nucl. Energy 113, 147-161.

Newton, T., Hosking, G., Hutton, L., Powney, D., Turland, B., Shuttleworth, E., 2008. Developments within WIMS10, in: Proc. PHYSOR 2008, Interlaken, Switzerland.

Otto, R.T., 2013. Core Optimization in a Thorium-based Civil Marine Propulsion Reactor. Master's thesis. University of Cambridge. Cambridge, UK.

Pint, B.A., Terrani, K.A., Brady, M.P., Cheng, T., Keiser, J.R., 2013. High temperature oxidation of fuel cladding candidate materials in steam-hydrogen environments. J. Nucl. Mater. 440, 420-427.

Pramuditya, S., Takahashi, M., 2013. Core design study for power uprating of integral primary system PWR. Ann. Nucl. Energy 59, 16-24.

Prasad, S., Abdulla, A., Morgan, M.G., Azevedo, I.L., 2015. Nonproliferation improvements and challenges presented by small modular reactors. Prog. Nucl. Energy 80, 102-109.

Ragheb, M., 2011. Nuclear naval propulsion, in: Tsvetkov, P. (Ed.), Nuclear Power - Deployment, Operation and Sustainability. InTech, Rijeka, Croatia. chapter 1, pp. 3-32.

Schinas, O., Stefanakos, C.N., 2012. Cost assessment of environmental regulation and options for marine operators. Transport. Res. C-Emer. 25, 81-99.

Shibata, K., Iwamoto, O., Nakagawa, T., Iwamoto, N., Ichihara, A., Kunieda, S., Chiba, S., Furutaka, K., Otuka, N., Ohasawa, T., 2011. JENDL-4.0: a new library for nuclear science and engineering. J. Nucl. Sci. Technol. 48, 1-30.

Shwageraus, E., Zhao, X., Driscoll, M.J., Hejzlar, P., Kazimi, M.S., Herring, J.S., 2004. Microheterogeneous thoria-urania fuels for pressurized water reactors. Nucl. Technol. 147, 20-36.

Sukjai, Y., Kazimi, M.S., 2015. Performance of thoria fuels and sic cladding for burning of plutonium in pressurized water reactors, in: Proc. Advances in Nuclear Fuel Management V (ANFM 2015), Hilton Head Island, SC, USA.

Terrani, K.A., Zinkle, J.S., Snead, L.L., 2014. Advanced oxidation-resistant iron-based alloys for LWR fuel cladding. J. Nucl. Mater. 448, 420-435.

Todosow, M., Galperin, A., Herring, S., Kazimi, M., Downar, T., Morozov, A., 2005. Use of thorium in light water reactors. Nucl. Technol. 151, 168-176.

Vergara, J.A., McKesson, C.B., 2002. Nuclear propulsion in high-performance cargo vessels. Mar. Technol. $39,1-11$.

Wu, X., Kozlowski, T., Hales, J.D., 2015. Neutronics and fuel performance evaluation of accident tolerant FeCrAl cladding under normal operation conditions. Ann. Nucl. Energy 85, 763-775.

Younker, I., Fratoni, M., 2016. Neutronic analysis of candidate accident-tolerant cladding concepts in PWR. Prog. Nucl. Energy 88, 10-18.

Zainuddin, N.Z., 2015. In-core Optimisation of Thorium-Plutonium-fuelled PWR Cores. Ph.D. thesis. University of Cambridge. Cambridge, UK. 
Zhao, X., 2001. Micro-heterogeneous Thorium Based Fuel Concepts for Pressurized Water Reactors. Ph.D. thesis. Cambridge, MA.

Zverev, D., Pakhomov, A., Polunichev, V., Veshnyakov, K., Kabin, S., 2013. RITM-200: new-generation reactor for a new nuclear icebreaker. At. Energ. 113, 404-409. 